ARTICLE

\title{
TDP-43 and PINK1 mediate CHCHD10S59L mutation-induced defects in Drosophila and in vitro
}

Minwoo Baek ${ }^{1,5}$, Yun-Jeong Choe ${ }^{1,5}$, Sylvie Bannwarth², JiHye Kim¹, Swati Maitra1, Gerald W. Dorn $\|^{3}$, J. Paul Taylor ${ }^{4}$, Veronique Paquis-Flucklinger ${ }^{2} \&$ Nam Chul Kim (D) $^{1 凶}$

Mutations in coiled-coil-helix-coiled-coil-helix domain containing 10 (CHCHD10) can cause amyotrophic lateral sclerosis and frontotemporal dementia (ALS-FTD). However, the underlying mechanisms are unclear. Here, we generate $\mathrm{CHCH} 10^{S 59 L}$-mutant Drosophila melanogaster and HeLa cell lines to model CHCHD10-associated ALS-FTD. The CHCHD10S59L mutation results in cell toxicity in several tissues and mitochondrial defects. $\mathrm{CHCHD10} 559 \mathrm{~L}$ independently affects the TDP-43 and PINK1 pathways. CHCHD10 559 expression increases TDP-43 insolubility and mitochondrial translocation. Blocking TDP-43 mitochondrial translocation with a peptide inhibitor reduced $\mathrm{CHCHD10} 559 \mathrm{~L}$-mediated toxicity. While genetic and pharmacological modulation of PINK1 expression and activity of its substrates rescues and mitigates the $\mathrm{CHCHD10} 559$-induced phenotypes and mitochondrial defects, respectively, in both Drosophila and HeLa cells. Our findings suggest that $\mathrm{CHCHD10} 55$ L-induced TDP-43 mitochondrial translocation and chronic activation of PINK1-mediated pathways result in dominant toxicity, providing a mechanistic insight into the $\mathrm{CHCHD10}$ mutations associated with ALS-FTD.

\footnotetext{
${ }^{1}$ Department of Pharmacy Practice and Pharmaceutical Sciences, College of Pharmacy, University of Minnesota, Duluth, MN, USA. ${ }^{2}$ Inserm U1081, CNRS UMR7284, IRCAN, Université Côte d'Azur, CHU de Nice, Nice, France. ${ }^{3}$ Center for Pharmacogenomics, Washington University School of Medicine, St. Louis, MO, USA. ${ }^{4}$ Howard Hughes Medical Institute and Department of Cell and Molecular Biology, St. Jude Children's Research Hospital, Memphis, TN, USA.

${ }^{5}$ These authors contributed equally: Minwoo Baek, Yun-Jeong Choe. ${ }^{凶}$ email: kimn@umn.edu
} 
n 2014, Bannwarth et al. ${ }^{1}$ identified an S59L substitution in coiled-coil-helix-coiled-coil-helix domain containing 10 (CHCHD10) as the causes of a familial disease characterized by motor function defects, declined cognitive function, and myopathy. Subsequent analyses revealed that $C H C H D 10^{S 59 L}$ is a genetic cause of amyotrophic lateral sclerosis with frontotemporal dementia (ALS-FTD) ${ }^{1}$. CHCHD10 encodes a functionally unknown, small protein comprising a putative $\mathrm{N}$-terminal mitochondrial-targeting sequence and a C-terminal $\mathrm{CHCHD}$ domain ${ }^{1}$. Identification of mutant $\mathrm{CHCHD} 10$ in ALS-FTD and related diseases and its predominant localization in mitochondria provide an opportunity to investigate the mitochondrial origin of neurodegenerative diseases.

Many additional $\mathrm{CHCHD} 10$ variants are now known to cause ALS, FTD, and other related degenerative diseases ${ }^{2,3}$. However, the pathogenicity and penetrance of some variants are debatable. Although $C H C H D 10^{R 15 L}$ was identified in familial and sporadic cases of ALS, the existence of unaffected carriers in familial cases suggests incomplete penetrance ${ }^{4-7}$. The $C H C H D 10^{P 34 S}$ variant occurs in sporadic ALS ${ }^{8,9}$, ALS-FTD ${ }^{10}$, Parkinson disease ${ }^{6}$, and Alzheimer's disease ${ }^{6}$, and its overexpression causes mitochondrial defects ${ }^{11}$. However, its pathogenicity is not well supported by genetic evidence ${ }^{12,13}$. CHCHD $10^{G 58 R}$ and CHCHD10 $666 \mathrm{~V}$ were identified in mitochondrial myopathy and late-onset spinal muscular atrophy, Jokela type (SMAJ), or Charcot-Marie-Tooth disease type 2 (CMT2), respectively ${ }^{5,14-16}$. Indeed, other ALSFTD-associated genes, such as valosin-containing protein (VCP) and Matrin 3 (MATR3), also exhibit clinical pleiotropy, including myopathy ${ }^{17,18}$. Thus, although neurodegenerative diseases may be generally associated with myopathy, the basis of CHCHD10mediated clinical pleiotropy is not understood.

The CHCHD10 mutations identified in familial diseases are dominantly inherited ${ }^{1,4,5,14}$. However, experimental evidence does not support that all disease-causing variants have the same mode of action. Bannwarth et al. ${ }^{1}$ showed that CHCHD10 expression in patient tissues is not affected and that overexpression of $\mathrm{CHCHD} 10^{S 59 \mathrm{~L}}$ causes mitochondrial defects similar to that in affected patients. This suggests that $\mathrm{CHCHD} 10^{S 59 L}$ is a dominant gain-of-function mutant. Woo et al. ${ }^{19}$ also confirmed such overexpression-mediated cell toxicity of $\mathrm{CHCHD} 10^{S 59 L}$. However, $\mathrm{CHCHD} 10^{S 59 L}$ does not retain its wild-type (WT)-like activity, indicative of a dominant-negative mechanism. Furthermore, patient fibroblasts with either $C H C H D 10^{R 15 L}$ or CHCHD $10^{G 66 V}$ exhibit reduced expression and protein instability, supporting a haploinsufficiency mechanism ${ }^{20,21}$. These data indicate that more detailed investigation is necessary to understand the disease-causing mechanism(s) of mutant CHCHD10 and suggest that $C H C H D 10$ mutations have multiple modes of action.

Although some molecular mechanisms for CHCHD10-mediated cell toxicity are known, it is unclear how these mechanisms drive the disease phenotype and whether they can be controlled therapeutically. CHCHD10 interacts with components of the mitochondrial contact site and cristae organizing system (MICOS), and the MICOS complex is decreased in patients with CHCHD10 mutations ${ }^{11}$. However, Straub et al. ${ }^{21}$ reported that CHCHD10 is not well localized with the MICOS complex and that $\mathrm{CHCHD} 10-\mathrm{CHCHD} 2$ hetero-complex formation decreases in patient fibroblasts carrying $C H C H D 10^{R 15 L}$. Although Woo et al. ${ }^{19}$ reported the physical interaction of CHCHD10 and TDP43 , Genin et al. $^{22}$ demonstrated that phosphorylated TDP-43 levels are not associated with the phenotypic severity of $\mathrm{CHCHD} 10^{S 59 L}$ or $\mathrm{CHCHD} 10^{\mathrm{G} 66 \mathrm{~V}}$. Indeed, severity was more closely associated with MICOS complex formation ${ }^{22}$. Because of a lack of a genetically tractable model, most studies rely on the analysis of protein-protein interactions. Therefore, systematic evaluation of which upstream effectors or signaling pathways are essential for disease pathogenesis is lacking.

Identification of $\mathrm{CHCHD} 10$ mutations and pathogenic mitochondrial pathways in ALS-FTD suggest that mitochondrial defects are a primary cause of ALS, FTD, or other related diseases $^{23-28}$. However, this also raises several intriguing questions. Are mitochondrial defects the primary driver of disease in specific subtypes of ALS-FTD? Do all of these subtypes share a common mechanism? Can mitochondrial defects in different subtypes be rescued by activating protective pathways or targeting a common pathogenic mechanism? To address these questions, we used Drosophila and mammalian cell culture models of CHCHD10 ${ }^{S 59 L}$-mediated cell toxicity. In these models, we found that $C H C H D 10^{S 59}$ expression imparts a toxic gain of function and that this dominant toxicity is mediated through two distinct axes: TDP-43 and PINK1. CHCHD10 $559 L$ expression increased insolubility and mitochondrial association of TDP-43 and also activated PINK1-mediated pathways. Pharmacologic treatments with peptide inhibitors of TDP-43 translocation to mitochondria or PINK1 kinase activity successfully mitigated degenerative phenotypes in HeLa cells. Small-molecule agonists of mitofusin (MFN), a downstream substrate of PINK1, rescued mutant CHCHD10-induced mitochondrial defects in Drosophila and HeLa cells. Moreover, the MFN agonists enhanced mitochondrial ATP production in a Drosophila ALS-FTD model expressing C9orf72 with expanded GGGGCC repeats. These findings suggest that $C H C H D 10^{S 59 L}$-induced ALS-FTD shares a disease-causing mechanism through mitochondrial translocation of TDP-43 with other subtypes of ALS-FTD and that modulating mitochondrial function by targeting PINK1-mediated pathways may provide a therapeutic strategy for $\mathrm{CHCHD} 10$-mediated disease.

\section{Results}

CHCHD2 and CHCHD10 share a common Drosophila melanogaster ortholog. To elucidate whether an ortholog of CHCHD10 exists in Drosophila, we used the Drosophila RNAi (RNA interference) Screening Center's Integrative Ortholog Prediction Tool ${ }^{29}$. We found that Drosophila and humans (Homo sapiens) have three and two homologous genes for CHCHD10, respectively. Further phylogenetic analysis with a neighborjoining tree of these genes revealed that CG5010 is the Drosophila gene sharing the highest homology with both human CHCHD2 and CHCHD10 (Supplementary Fig. 1a). Two additional Drosophila homologs, Dmel $\backslash C G 31007$ and Dmel $\backslash C G 31008$ appeared independently after their speciation. The substituted amino acids in human patients are conserved in Drosophila CG5010 (Fig. 1a). In addition, CG5010 is highly expressed in all Drosophila tissues, whereas Dmel \CG31007 and Dmel\CG31008 are expressed only in the testis and weakly in the imaginal discs. A comparison between $H$. sapiens and Mus musculus suggested that $\mathrm{CHCHD} 10$ and $\mathrm{CHCHD} 2$ were duplicated before their speciation and that they may be involved in common processes, but in a distinct manner ${ }^{30}$. According to its phylogenetic and expression profile, we deemed CG5010 as a common Drosophila ortholog for both CHCHD10 and CHCHD2. Therefore, we hereafter refer to $\mathrm{CG} 5010$ as $\mathrm{C} 2 \mathrm{C} 10 \mathrm{H}$ (i.e., $\mathrm{CHCHD} 2$ and CHCHD10 homolog) and Dmel $\backslash C G 31007$ and Dmel $\backslash C G 31008$ as C2C10L1 (i.e., CHCHD2-CHCHD10-like 1) and C2C10L2 (i.e., CHCHD2 and CHDHD10-like 2), respectively.

CHCHD10 ${ }^{\text {S59L }}$ causes dominant degeneration in fly eyes. To develop Drosophila models for mutant CHCHD10-induced human disease, we generated both Drosophila and codonoptimized human versions of transgenic animals carrying $C 2 \mathrm{C} \mathrm{H}^{W T}$ and S81L (CHCHD10 ${ }^{W T}$ and S59L in human), with 
a

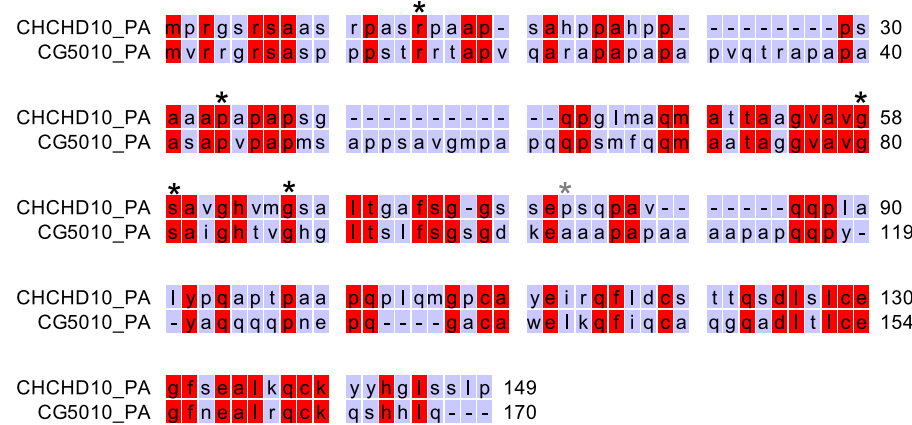

d

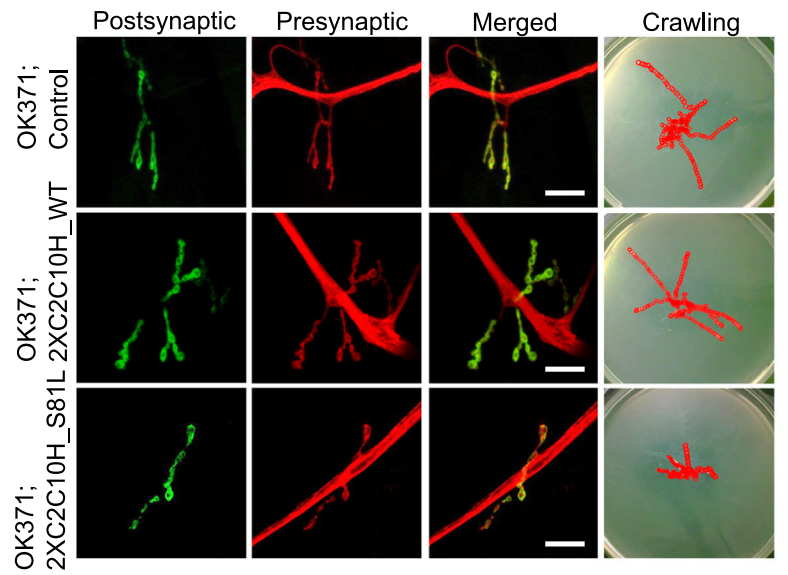

b

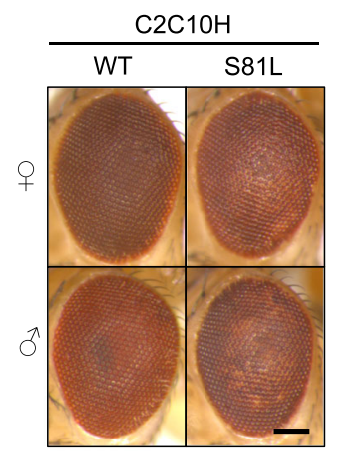

C

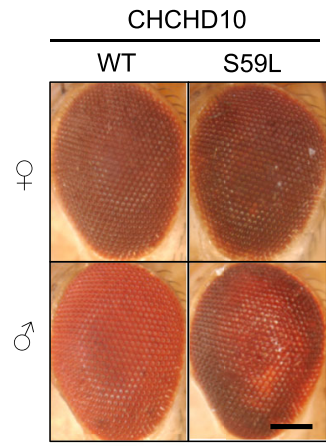

e

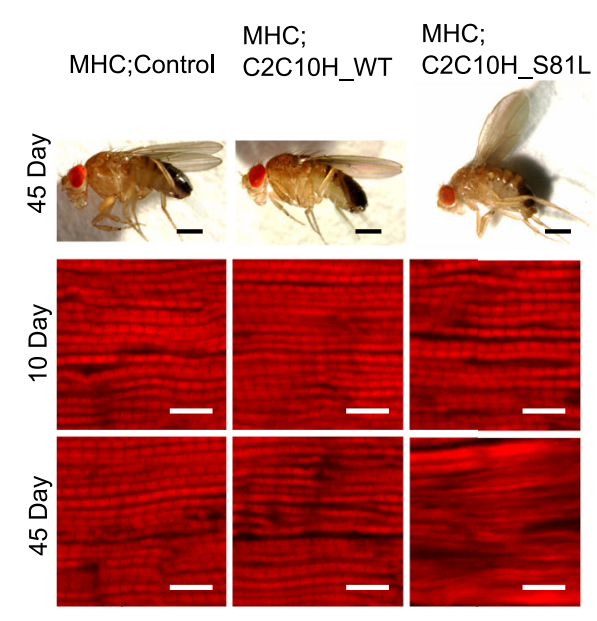

g

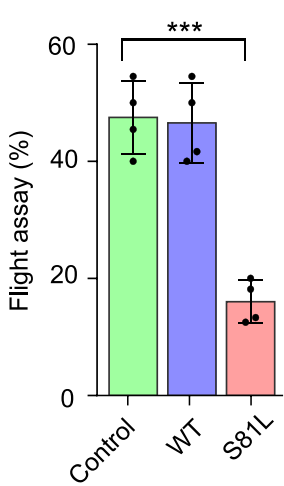

f

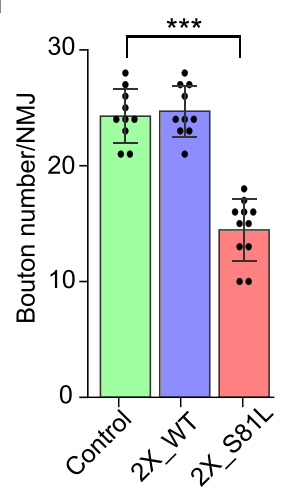

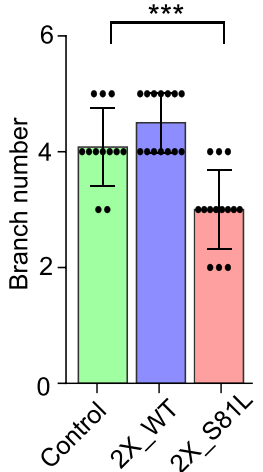

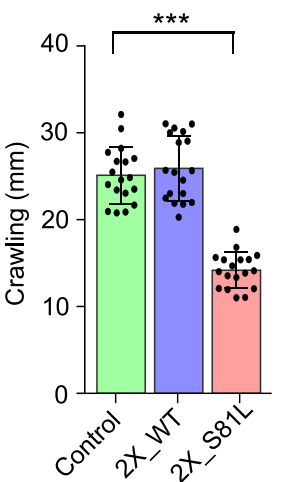

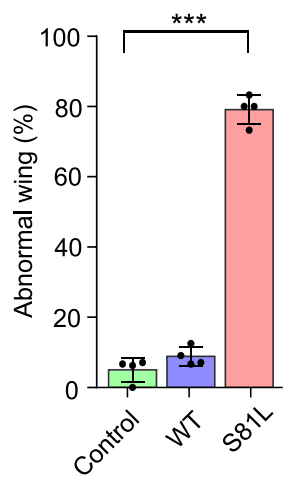

Fig. $1 \mathrm{C2C10H}^{\mathbf{8 8 1 L}}$ is toxic in Drosophila eyes, neurons, and muscles. a Protein sequence alignment of human $\mathrm{CHCHD10}$ and Drosophila $\mathrm{C} 2 \mathrm{C} 10 \mathrm{H}$ (CG5010). Disease-causing sites (asterisk) are conserved between human $\mathrm{CHCHD} 10$ and Drosophila $\mathrm{C} 2 \mathrm{C} 10 \mathrm{H}$. b $\mathrm{C}_{2} \mathrm{C} 10 \mathrm{H}^{581 \mathrm{~L}}$ (representative images from two independent experiments) and $\mathbf{c}$ human $\mathrm{CHCHD10} 559 \mathrm{~L}$ cause age-dependent mild rough eye phenotypes in 40-day-old flies (representative images from two independent experiments). Scale bar $=200 \mu \mathrm{m}$. d Representative images of neuromuscular junctions and crawling traces from the genotypes indicated (see below for statistical analysis). Scale bar $=20 \mu \mathrm{m}$. e Adult thoraxes dissected to expose longitudinal indirect flight muscles and stained with

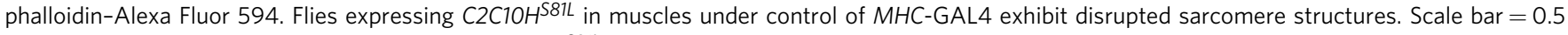

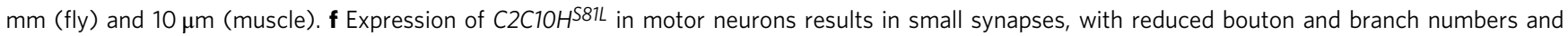
defective locomotive activity assessed by the crawling behavior of third-instar larvae. Data are mean \pm SD (one-way ANOVA and post hoc Dunnett test, two-sided, ${ }^{\star \star \star} p=5.3 \mathrm{e}-10,0.0002$ and $3.8 \mathrm{e}-14$ for bouton number $(n=10-11)$, branch number $(n=12-14)$, and crawling distance $(n=18)$ from three independent experiments, respectively). $\mathbf{g}$ Expression of $\mathrm{C}_{2} \mathrm{C} 10 \mathrm{H}^{\mathrm{S} 81 \mathrm{~L}}$ in muscle tissues causes abnormal wing postures and locomotor defects assessed by flight ability. Data are mean \pm SD (one-way ANOVA and post hoc Dunnett test, two-sided, ${ }^{\star \star \star} p=4.3 \mathrm{e}-10,5.5 \mathrm{e}-05$ for abnormal wing posture, flight assay, respectively; $n=4$ with $>40$ flies). 
or without a C-terminal FLAG tag by $Ф$ C31 integrase-mediated site-specific integration into the attp2 landing site on the third chromosome.

When $\mathrm{C} 2 \mathrm{C} 10 \mathrm{H}^{W T}$ and $\mathrm{CHCHD} 10^{W T}$ were expressed in Drosophila eyes by the glass multimer reporter (GMR)-GAL4 driver, they did not cause any abnormal phenotypes at eclosion. Although $\mathrm{C} 2 \mathrm{C} 10 \mathrm{H}^{W T}$ and $\mathrm{CHCHD} 10^{W T}$ expression did not cause any apparent defects, expression of $C 2 \mathrm{C}_{10 H} \mathrm{H}^{S 81 L}$ and CHCHD $10^{S 59 L}$ caused mild but mutation-dependent degeneration with depigmentation as the flies aged, regardless of FLAG tagging (Fig. 1b, c and Supplementary Fig. 1d). The severity of rough eye phenotypes was determined by Flynotyper scoring of the disorderliness of ommatidia ${ }^{31}$. There were statistically significant differences in eye phenotypes (Supplementary Fig. 1b, c, d) without substantial differences in the expression level of WT and mutant proteins (Supplementary Fig. 1e). When we generated fly lines carrying two copies of $\mathrm{C} 2 \mathrm{C} 10 \mathrm{H}^{\mathrm{S} 81 \mathrm{~L}}$ at the attp2 locus with GMR-GAL4, the rough eye phenotype of $\mathrm{C} 2 \mathrm{C} 10 \mathrm{H}^{S 81 L}$ was enhanced and obvious at eclosion due to high levels of expression by transvection ${ }^{32}$ (Supplementary Fig. 1f, g). However, the expression of two copies of $\mathrm{C} 2 \mathrm{C} 10 \mathrm{H}^{W T}$ did not induce any eye defects (Supplementary Fig. 1f). These findings indicate that overexpression of $\mathrm{C}^{\mathrm{C}} 10 \mathrm{H}^{\mathrm{S} 81 \mathrm{~L}}\left(\mathrm{CHCHD10} \mathrm{C}^{S 9 L}\right)$ generates mutant-dependent degeneration in Drosophila eyes.

$\mathrm{C} 2 \mathrm{C} 10 \mathrm{H}^{\mathrm{S} 81 \mathrm{~L}}$ recapitulates morphologic and functional defects in motor neurons and muscles. Patients carrying $\mathrm{CHCHD} 10^{S 59 \mathrm{~L}}$ experience motor neuron defects and myopathy. Thus, we examined whether $\mathrm{C} 2 \mathrm{C} 10 \mathrm{H}^{S 81 L}$ and $\mathrm{CHCHD} 10^{S 59 L}$ expression cause degenerative phenotypes in Drosophila motor neurons and muscles. Due to the weaker cell toxicity of $\mathrm{CHCHD} 10^{\mathrm{S} 59 \mathrm{~L}} \mathrm{com}-$ pared to $\mathrm{C} 2 \mathrm{C} 10 \mathrm{H}^{\mathrm{S} 81 \mathrm{~L}}$ in the Drosophila system, $\mathrm{C} 2 \mathrm{C} 10 \mathrm{H}^{\mathrm{S} 81 \mathrm{~L}}$ was mainly used in most experiments. Expressing $\mathrm{C} 2 \mathrm{C} 10 \mathrm{H}^{W T}$ or $C 2 \mathrm{C}_{10 H} \mathrm{H}^{S 1 L}$ in motor neurons with OK371-GAL4 did not cause noticeable abnormalities in larvae or adult flies, including viability and fertility. However, homozygous animals for OK371-GAL4 and $C 2 \mathrm{C}_{10 H^{S 81 L}}$ exhibited robust degenerative phenotypes, whereas homozygous $\mathrm{C} 2 \mathrm{C} 10 \mathrm{H}^{W T}$ flies with $\mathrm{OK} 371-\mathrm{GAL} 4 \mathrm{did}$ not show any abnormalities. Third-instar $\mathrm{C} 2 \mathrm{C}_{10 \mathrm{H}^{S 81 L}}$ homozygous larvae showed striking locomotor dysfunction (Fig. 1d, f), with marked morphologic defects in their neuromuscular junctions (NMJs), including decreased synaptic bouton and branch numbers, and the absence of such NMJ defects in driver-only or $C 2 \mathrm{C}_{10 H^{W T}}$-expressing flies (Fig. 1d, f). Consistently, a locomotive defect was also observed with third-instar homozygous larvae expressing $\mathrm{CHCHD} 10^{\mathrm{S} 59 \mathrm{~L}}$ in motor neurons (Supplementary Fig. 1h). Expression of $C 2 C 10 H^{S 81 L}$ but not $C 2 C 10 H^{W T}$ in indirect flight muscles with $M H C$-GAL4 led to mutant-dependent muscle degeneration, which was characterized by abnormal wing posture and loss of sarcomere architecture in aged flies (Fig. 1e, g). In addition, $C 2 \mathrm{C} 0 \mathrm{H}^{S 81 L}$ expression in muscles resulted in functional locomotor defects, as evident in flight assays (Fig. 1g). These findings reveal that the Drosophila model recapitulates the dominant cell toxicity of $C H C H D 10^{S 59 L}$ in vivo.

CHCHD10 $^{\text {S59L }}$ induces mitochondrial defects. CHCHD10 is primarily localized at cristae junctions in the mitochondrial intermembrane space ${ }^{1,33}$. To determine whether $C H C H D 10^{S 59 L}$ induces mitochondrial defects, we transiently expressed CHCHD $10^{W T}$ or $C H C H D 10^{S 59 L}$ tagged with C-terminal FLAG in HeLa cells. Immunocytochemistry using an anti-FLAG antibody revealed $\mathrm{CHCHD} 10$ localization in mitochondria but not in other cellular sites (Fig. 2a). Consistent with the previous reports ${ }^{1,19,34}$, expression of $\mathrm{CHCHD} 10^{S 59 \mathrm{~L}}$ caused notable mitochondrial fragmentation and functional respiratory defects, as measured by
Seahorse XF Cell Mito Stress tests. In contrast, $\mathrm{CHCHD10}{ }^{W T}$ expressed at a similar level to $\mathrm{CHCHD} 10^{S 59 L}$, induced mitochondrial elongation and improved respiratory function over that of empty vector-transfected cells (Fig. 2a-c and Supplementary Fig. 2a). $\mathrm{CHCHD} 10^{\mathrm{S} 59 \mathrm{~L}}$ displayed a punctate staining pattern in mitochondria, whereas CHCHD10WT staining was evenly distributed (Fig. 2a, b). It is unclear whether this punctate pattern is due to CHCHD10 aggregation or mitochondrial fragmentation because the staining patterns of the mitochondrial marker and CHCHD $10^{\text {S59L }}$ mostly overlapped (Fig. 2a, b).

To examine mitochondrial morphology and function in response to $\mathrm{C} 2 \mathrm{C} 10 \mathrm{H}^{S 81 L}$ expression in Drosophila, we expressed two copies of $C 2 \mathrm{C} 0 \mathrm{H}^{S 81 L}$ in muscle tissues with the MHC-GAL4 driver. Visualization of mitochondria with a green fluorophoreconjugated streptavidin revealed that expression of $\mathrm{C} 2 \mathrm{C} 10 \mathrm{H}^{S 81 L}$ in indirect flight muscles resulted in muscular degeneration, along with fragmented mitochondria. This finding was in contrast with our observations in the indirect flight muscles of MHC-GAL4 only or $\mathrm{C} 2 \mathrm{C} 10 \mathrm{H}^{W T}$-expressing flies (Fig. 2d). We measured ATP levels as an indicator of mitochondrial dysfunction in the flies' thoraxes, which contain primarily muscle tissues. We observed reduced ATP levels in the muscle tissues of flies expressing $\mathrm{C} 2 \mathrm{C}_{10 H^{S 81 L}}$, as compared with those of $\mathrm{MHC}$-GAL4/+ control flies (Fig. 2e). Consistent with the effect of $\mathrm{CHCHD10}$ WT overexpression in HeLa cells, C2C10H ${ }^{W T}$ expression also increased ATP levels in Drosophila muscle tissues (Fig. 2e). Consistently, expression of CHCHD10 ${ }^{\mathrm{S} 59 \mathrm{~L}}$ protein in Drosophila muscles caused similar defects (Supplementary Fig. 2d). Therefore, $\mathrm{C} 2 \mathrm{C} 10 \mathrm{H}^{S 81 L}$ and $\mathrm{CHCHD} 10^{S 59 L}$ induced consistent mitochondrial fragmentation and functional respiratory defects in Drosophila and mammalian cells.

CHCHD10 $559 \mathrm{~L}$ is not a hypermorphic gain-of-function mutant. The S59L substitution in CHCHD10 is dominantly inherited $^{1}$. The dominant cell toxicity imparted by $\mathrm{CHCHD} 10^{S 59 L}$ overexpression suggests two possible modes of action: dominant negative or dominant gain of function. There are also two possible modes for gain of function: gain of a WT function (hypermorph) or gain of an abnormal function (neomorph) ${ }^{35}$. First, we tested if $C H C H D 10^{S 59 L}$ is a hypermorph. If $C H C H D 10^{S 59 L}$ enhances its own functions to generate cell toxicity, $\mathrm{CHCHD} 10^{W T}$ co-expression with $\mathrm{CHCHD} 10^{\mathrm{S} 59 \mathrm{~L}}$ should enhance the degenerative phenotype of $\mathrm{CHCHD} 10^{\mathrm{S} 59 \mathrm{~L}}$. For this genetic interaction study using $\mathrm{C} 2 \mathrm{C} 10 \mathrm{H}^{S 81 L}$, we generated a fly model carrying two copies of $C 2 \mathrm{C} 0 \mathrm{H}^{S 81 L}$ in the second and the third chromosomes. When the two $C 2 C 10 H^{S 81 L}$ copies were expressed via GMRGAL4, it induced relatively mild rough eye phenotypes at eclosion (Fig. 3a) compared with that of third chromosome homozygotes (Supplementary Fig. 1f). We tested several different control Drosophila lines with this model fly and did not observe the significant modification of phenotypes by the control lines (Supplementary Fig. 3a). Significantly, C2C1OH ${ }^{W T}$ co-expression did not enhance but suppressed the $C 2 C 10 H^{S 81 L}$-induced rough eye phenotype (Fig. 3a), in addition to the morphologic and functional mitochondrial defects in both Drosophila (Fig. 3b, c) and HeLa cells (Fig. 3d, e and Supplementary Fig. 3c). $\mathrm{C} 2 \mathrm{C} 10 \mathrm{H}^{S 81 L_{\text {-induced }}}$ rough eye phenotypes were also not enhanced but rescued by human $\mathrm{CHCHD} 10^{W T}$ co-expression (Supplementary Fig. 3e). This suggests that $\mathrm{CHCHD10^{S59L }}$ is not a hypermorph enhancing its own WT activity. To exclude the possibility of unknown positional effects such as transvection, we generated another transgenic fly line with $\mathrm{C} 2 \mathrm{C}_{10 H^{W T}}$ or $\mathrm{C} 2 \mathrm{C} 10 \mathrm{H}^{S 81 L}$ in an unrelated landing site, VK27. C2C1OH ${ }^{W T}{ }_{V K 27}$ also rescued the $C 2 \mathrm{C}_{10 H^{S 81 L}}$ phenotypes, whereas $\mathrm{C}^{2} \mathrm{C}_{10 H^{S 81 L}}{ }_{V K 27}$ enhanced the rough eye phenotype 
a

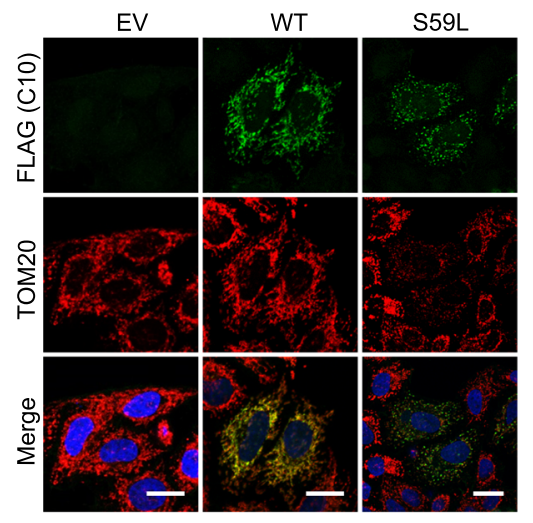

b

Mitochondria \& 10 signal length

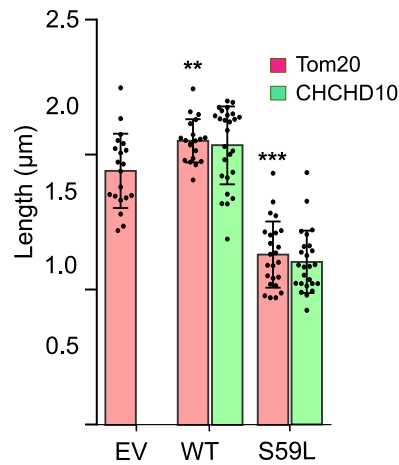

C

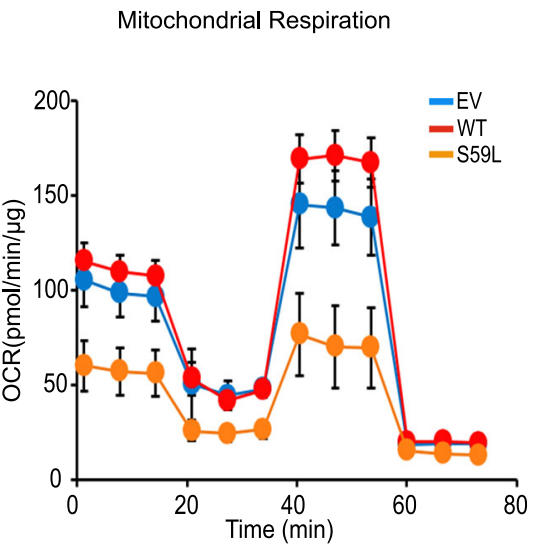

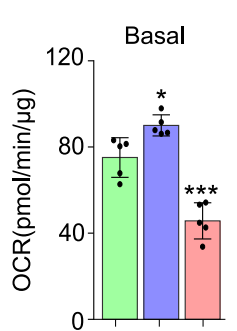
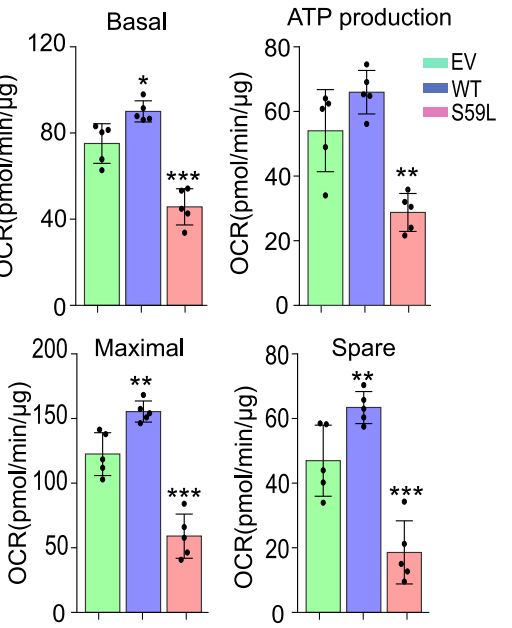

d
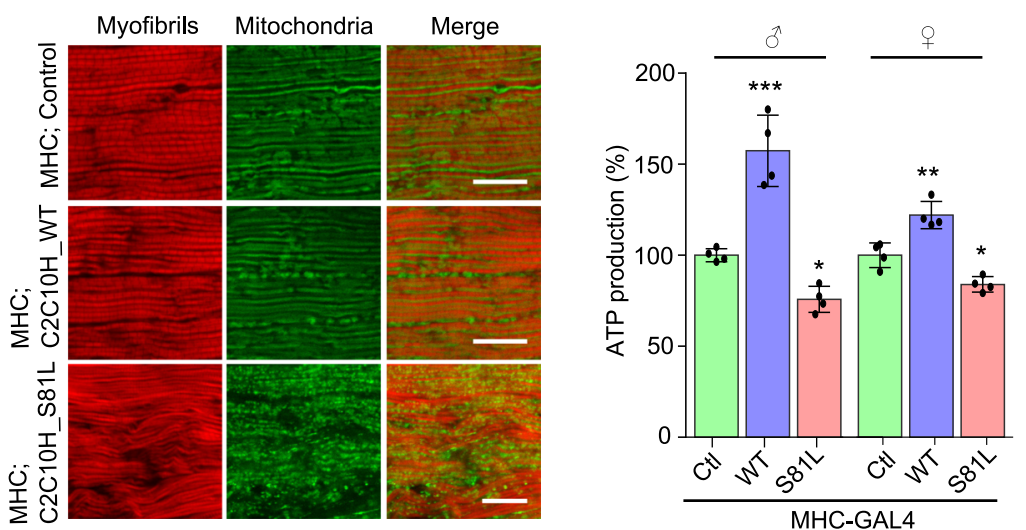

Fig. 2 CHCHD10 $559 L$ mutant induces mitochondrial defects. HeLa cells were transfected with FLAG-tagged CHCHD10WT, CHCHD10 $559 \mathrm{~L}$, and empty vector (EV). a Representative images from three independent experiments of HeLa cells immunostained with antibodies against FLAG (green, transfected CHCHD10) and TOM20 (red, mitochondria) $24 \mathrm{~h}$ after transfection. Scale bar $=20 \mu \mathrm{m}$. b Quantification of CHCHD10-FLAG (green) and TOM20 (red) signal length. Data are shown as mean \pm SD (one-way ANOVA and post hoc Dunnett test, two-sided, comparison with $E V$, ${ }^{\star \star} p=0.0069$, ${ }^{\star \star \star} p=3.7 e-12$; $n=20-24$ cells from three independent experiments). c Oxygen consumption rate (OCR) for each transfectant was measured. After measuring basal respiration rates, oligomycin, FCCP, and antimycin A/rotenone were serially injected to measure ATP production and maximal respiration, respectively. Spare respiratory capacity was then calculated. Graphs of a single representative experiment are shown (mean \pm SD). Actual statistical analyses were performed with 11 independent experiments (one-way ANOVA and post hoc Dunnett test, two-sided, comparison with EV, ${ }^{\star} p=0.01867$ and ${ }^{\star \star \star} p=$ 0.00012 in basal level; ${ }^{\star \star} p=0.0015$ in ATP level; ${ }^{\star \star} p=0.0071$ and ${ }^{\star \star \star} p=3.2 \mathrm{e}-05$ in maximal level; ${ }^{\star \star} p=0.002453$ and ${ }^{\star \star \star} p=0.00058$ in spare capacity; detailed information on statistical analyses is available in Supplementary Fig. 9a). d Mitochondrial morphology of 45-day-old C2C10HWT- and

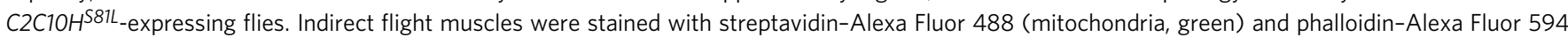
(actin filaments, red). Representative images from two independent experiments with 16 flies. Scale bar $=20 \mu \mathrm{m}$. e ATP levels in thoraxes containing muscle tissues of the indicated genotypes (aged 10 days) were measured. Data are shown as mean \pm SD (one-way ANOVA and post hoc Dunnett test, two-sided, ${ }^{\star \star *} p=0.00018$ and ${ }^{*} p=0.03662$ in male; ${ }^{\star \star} p=0.0015$ and ${ }^{*} p=0.011$ in female; $n=4$ independent experiments).

(Supplementary Fig. 3d). Consistent with our observations in Drosophila eyes, co-expression of $\mathrm{C} 2 \mathrm{C} 10 \mathrm{H}^{W T}$ mitigated the

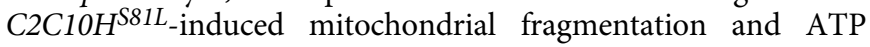
production defects in indirect flight muscles (Fig. 3b, c). An additional copy of $\mathrm{C} 2 \mathrm{C} 10 \mathrm{H}^{S 81 L}$ exacerbated the defects in both the eyes and muscles (Fig. $3 \mathrm{a}-\mathrm{c}$ ).

In HeLa cells, co-expression of CHCHD10WT with CHCHD $10^{S 59 L}$ clearly mitigated morphologic (Fig. 3d, e) and functional defects (Supplementary Fig. 3c). However, the punctate staining pattern of $\mathrm{CHCHD} 10^{\mathrm{S} 59 \mathrm{~L}}$ was not altered by CHCHD10 ${ }^{W T}$ co-expression (Fig. 3d, e), indicating that CHCHD $10^{W T}$ improves mitochondrial integrity by a mechanism other than restoring mutant protein aggregation. Immunostaining of indirect flight muscles revealed that $\mathrm{C} 2 \mathrm{C} 10 \mathrm{H}^{\mathrm{S} 81 \mathrm{~L}}$ and
$\mathrm{CHCHD} 10^{\mathrm{S} 59 \mathrm{~L}}$ proteins also formed aggregate-like structures in vivo (Supplementary Fig. 2b, c). Together, these results suggest that $C 2 C 10 H^{S 81 L}$ and $C H C H D 10^{S 59 L}$ clearly form aggregates and do not act as a hypermorph (gain of WT function).

CHCHD $10^{\text {S59L }}$ is a dominant gain-of-function mutant. The mislocalized punctate pattern of $\mathrm{CHCHD} 10^{\mathrm{S} 59 \mathrm{~L}}\left(\mathrm{C} 2 \mathrm{C} 10 \mathrm{H}^{\mathrm{S} 81 \mathrm{~L}}\right)$ suggests that $\mathrm{CHCHD} 10^{S 59 L}\left(\mathrm{C} 2 \mathrm{C} \mathrm{H}^{\mathrm{S} 81 L}\right)$ may be a dominant gain-of-toxic (neomorphic) mutant acquiring abnormal functions. To distinguish a dominant gain-of-toxic mutant from a dominant-negative mutant suppressing the activity of its WT form, we tested whether the toxicity of $C H C H D 10^{S 59 L}$ and $\mathrm{C} 2 \mathrm{C} 10 \mathrm{H}^{S 81 L}$ is dependent on the existence of $\mathrm{CHCHD} 10^{W T}$ and 
a

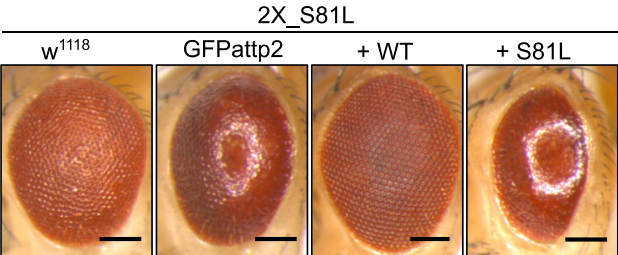

b

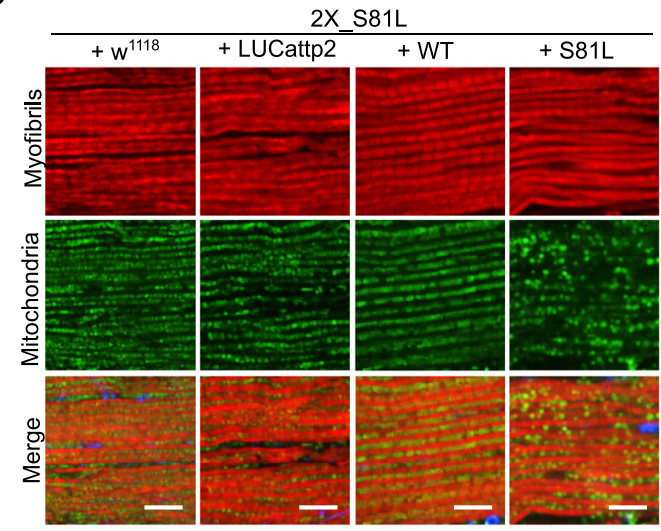

e

Mitochondrial length \& C10 signal length

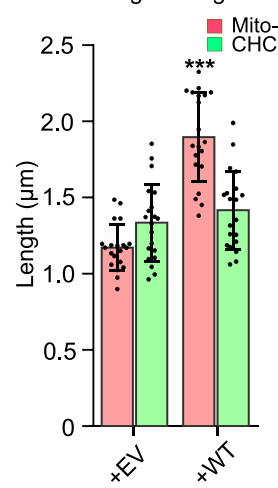

h

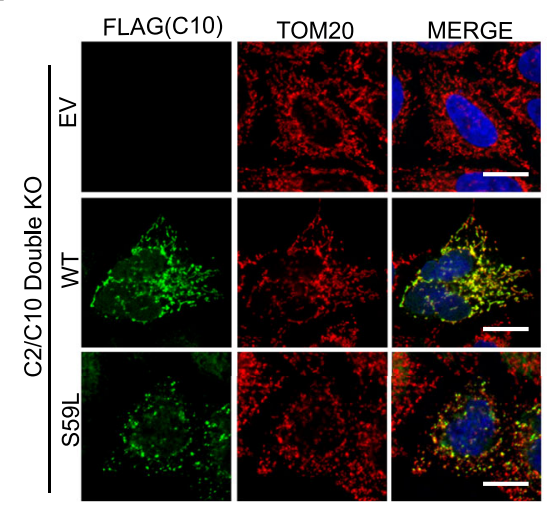

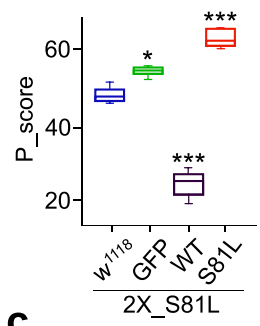

C

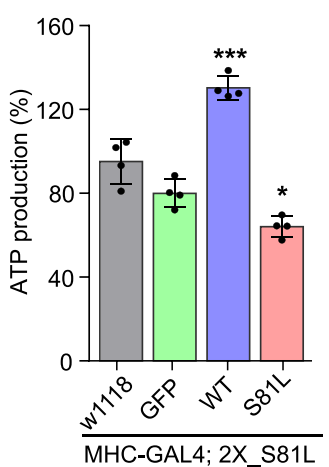

d

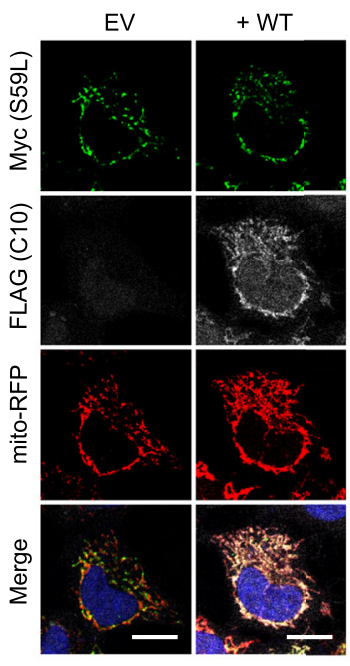

f
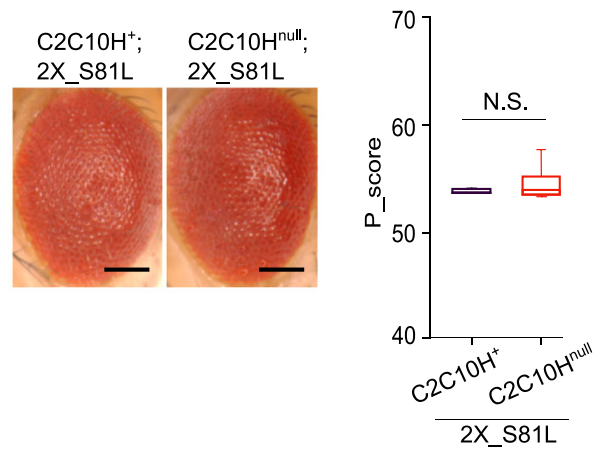

g

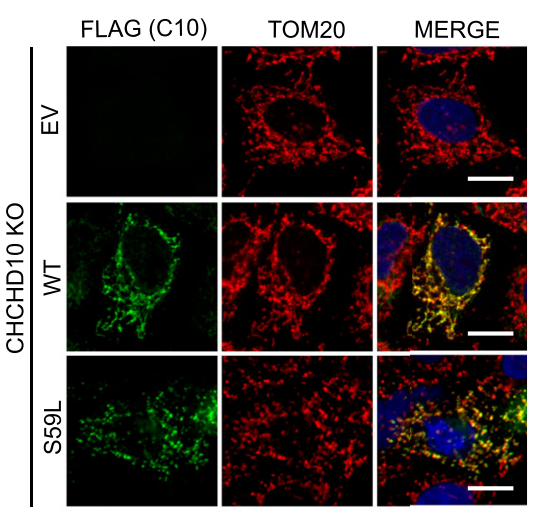

j

Mitochondrial length (TOM20)

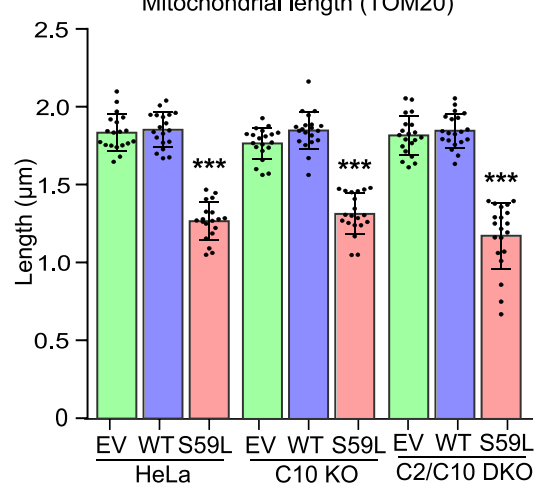

$\mathrm{C} 2 \mathrm{C} 10 \mathrm{H}^{W T}$, respectively. If they are true dominant negative, the phenotype of $\mathrm{C} 2 \mathrm{C} 10 \mathrm{H}$ deletion mutant $\left(\mathrm{C}^{2} \mathrm{C} 0 \mathrm{H}^{\text {null }}\right)$ animals and CHCHD10 knockout $\left(C H C H D 10^{K O}\right)$ cells will not be enhanced by the expression of $\mathrm{C}_{2} \mathrm{C}_{10 H^{81 L}}$ and $\mathrm{CHCHD} 10^{S 59 L}$. Because $\mathrm{C}^{2} \mathrm{C}_{10 H^{\text {null }}}$ flies do not exhibit an abnormal eye phenotype and are generally healthy ${ }^{36}$, we expected that the $\mathrm{C} 2 \mathrm{C} 10 \mathrm{H}^{S 81 L_{-}}$ induced rough eye phenotype would not be present in the $\mathrm{C}_{2} \mathrm{C}_{10 H^{\text {null }}}$ background if it is a dominant-negative mutant. However, the rough eye phenotype was robust in the $\mathrm{C}_{2} \mathrm{C}_{10 H^{n u l l}}$ background (Fig. 3f). Although we tested if two paralogs of C2C10H (Dmel $\mid C G 31007$ and Dmel lCG31008) can modify the $\mathrm{C} 2 \mathrm{C}_{10 H^{S 81 L}}$-induced phenotypes by RNAi-mediated knockdown 
Fig. 3 CHCHD10 $559 L$ shows gain-of-toxic function. a Expression of $\mathrm{C2C1OHWT}$ with two copies of $\mathrm{C} 2 \mathrm{C} 10 \mathrm{H}^{581 L}$ by GMR-GAL4 did not exacerbate $\mathrm{C}^{2} \mathrm{C} \mathrm{H}^{581 L}$-induced rough eye phenotypes. The severity of eye phenotypes was analyzed with Flynotyper ${ }^{31}$ after being processed by ilastik ${ }^{73}$. Boxes indicate median and 25th and 75th percentiles (one-way ANOVA and post hoc Dunnett test, two-sided, ${ }^{\star} p=0.00132,{ }^{\star \star \star} p<2 e-16$ for WT, and ${ }^{\star \star \star} p=$ $4 \mathrm{e}-06$ for S81L; $n=11,9,9$, and 5 for w $\mathrm{W}^{1118}$, GFP, WT, and S81L, respectively. Samples were collected from two to three independent experiments). Scale

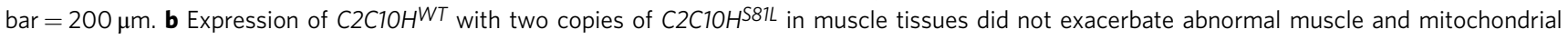
phenotypes. Scale bar $=10 \mu \mathrm{m}$. c ATP levels in each of the indicated genotypes were measured. Data are shown as mean \pm SD (one-way ANOVA and post hoc Dunnett test, two-sided, ${ }^{\star * \star} p=2.2 \mathrm{e}-07$ for $\mathrm{W}$ and ${ }^{\star} p=0.0234$ for $\mathrm{S} 81 \mathrm{~L} ; n=4$ independent experiments). d HeLa cells were co-transfected with Myc-tagged CHCHD10 $559 \mathrm{~L}$ and FLAG-tagged CHCHD10WT. Empty vector (EV) was used as a control. mTagRFP-T-Mito-7 was co-transfected to visualize mitochondria. Representative images of HeLa cells stained with anti-Myc (green) and anti-FLAG (gray) antibodies $24 \mathrm{~h}$ after transfection. Scale bar $=20 \mu \mathrm{m}$. e Quantification of mTagRFP-T-Mito-7 (mitochondria) and CHCHD10S59L-Myc signal length. Data are shown are mean \pm SD (two-sided $t$ test, ${ }^{\star \star \star} p=5.308 \mathrm{e}-12 ; n=20$ cells from three independent experiments). $\mathbf{f} \mathrm{C}^{\mathrm{C}} \mathrm{C} 10 \mathrm{H}^{581 L}$-induced rough eye phenotypes were robust in the $\mathrm{C} 2 \mathrm{C} 10 \mathrm{H}^{\text {null }}$ background. Boxes indicate median and 25th and 75th percentiles (two-sided $t$ test, $n=5$ flies from two independent experiments). Scale bar $=200 \mu$ m. g Representative images of $\mathrm{CHCHD10}{ }^{K O}$ HeLa cells transfected with $\mathrm{CHCHD10}$ WT and CHCHD10S59L. Cells were immunostained with antibodies against FLAG (green, CHCHD10) and TOM2O (red, mitochondria). Scale bar $=20 \mu \mathrm{m}$. h Representative images of CHCHD2 and $10^{\mathrm{KO}} \mathrm{HeLa}$ cells transfected with $\mathrm{CHCHD10}{ }^{W T}$ and $\mathrm{CHCHD10S59L}$. Cells were immunostained with FLAG (green, CHCHD10) and TOM2O (red, mitochondria) antibodies. Scale bar $=20 \mu \mathrm{m}$. i Quantification of TOM2O signal length (mitochondria) from CHCHD10WT and CHCHD10 S59L transfected HeLa, CHCHD10KO, and CHCHD2/10DKO HeLa. Data are shown as mean \pm SD (one-way ANOVA and post hoc Dunnett test, two-sided, ${ }^{\star \star *} p=3.3 \mathrm{e}-16,<2 \mathrm{e}-16$, and $<2 \mathrm{e}-16$ for HeLa, C10 KO and $\mathrm{C} 2 / \mathrm{C} 10 \mathrm{KO}$, respectively; $n=20$ cells from three independent experiments). $\mathbf{j}$ CHCHD10KO and CHCHD2/10DKO HeLa cells were transfected with EV, FLAG-tagged CHCHD10WT, and CHCHD10 $559 \mathrm{~L}$. After $24 \mathrm{~h}$, mitochondrial respiration for each transfectant was measured via Seahorse XF Cell Mito Stress tests. A representative graph of a single experiment is shown (mean $\pm \mathrm{SD}$ ). Actual statistical analyses were performed with three (C10 KO) and five (C2/ $\mathrm{C} 10 \mathrm{KO}$ ) independent experiments (one-way ANOVA and post hoc Dunnett test, two-sided, comparison with $\mathrm{EV}^{\star \star *} p=0.0073$ and ${ }^{\star \star \star} p=2.2 \mathrm{e}-06$ for WT and S59L in C10 KO, ${ }^{\star} p=0.0238$ and ${ }^{\star \star \star} p=2.1 \mathrm{e}-05$ for WT and S59L in C2/C10 KO; detailed information on statistical analyses is available in Supplementary Fig. 9b, c). NS not significant.

(Supplementary Table 1), we did not observe any significant interaction as they are not well expressed in Drosophila tissues except testis. Together, these results suggest that $C 2 C 10 H^{S 81 L}$ is not a dominant-negative mutant requiring $\mathrm{C} 2 \mathrm{C} 10 \mathrm{H}$ to generate its cell toxicity.

To further validate this result in a mammalian system, we generated $C H C H D 10^{K O}$ HeLa cells via the CRISPR/Cas9 system (Supplementary Fig. 3f). Although the CHCHD10 protein was not detected with an anti-CHCHD10 antibody in the $C H C H D 10^{K O}$ lines, mitochondrial morphology and respiratory functions were not affected (Fig. 3g, i and Supplementary Fig. 3g). Consistent with results from our Drosophila model, the expression of $C H C H D 10^{S 59 L}$ induced CDCDD10 ${ }^{W T}$-independent mitochondrial toxicity in $C H C H D 10^{K O}$ HeLa cells (Fig. $3 g, i, j$ and Supplementary Fig. 3h). To further investigate the possibility that $\mathrm{CHCHD} 10^{\text {S59L }}$ might suppress a close paralog CHCHD2 activity to generate the mitochondrial toxicity in a dominant-negative manner, we generated $\mathrm{CHCHD} 2$ and $\mathrm{CHCHD} 10$ doubleknockout $\left(C H C H D 2 / 10^{D K O}\right.$ ) cells (Supplementary Fig. 3f). Consistent with a previous report ${ }^{34}$, mitochondrial morphology and respiratory functions were not affected in $C H C H D 2 / 10^{D K O}$ (Fig. 3h, i and Supplementary Fig. 3g). The expression of CHCHD $10^{S 59 L}$ in CHCHD2/10 DKO cells also generated significant morphological and functional defects in mitochondria (Fig. $3 \mathrm{~h}-\mathrm{j}$ and Supplementary Fig. 3h). All these data support that CHCHD $10^{S 59 L}$ mutation is a dominant gain-of-function (i.e., toxic) mutation causing aggregation of the mutated protein, not a dominant-negative mutant suppressing the activity of its WT counterpart.

CHCHD10 $559 \mathrm{~L}$ proteins form insoluble aggregates. The punctate staining pattern of $\mathrm{CHCHD} 10^{\mathrm{S} 59 \mathrm{~L}}$ was not affected when $\mathrm{CHCHD} 10^{\mathrm{WT}}$ rescued $\mathrm{CHCHD} 10^{\mathrm{S} 59 \mathrm{~L}}$-induced mitochondrial fragmentation. The S59L substitution is located in the hydrophobic domain of an intrinsically disordered region in CHCHD10 (Supplementary Fig. 4a). Because the hydrophobic domain and intrinsically disordered region play a role in protein folding and aggregation, we examined whether $\mathrm{CHCHD} 10^{\text {S59L }}$ proteins accumulate in mitochondria as relatively insoluble aggregates. We detected insoluble $\mathrm{CHCHD} 10^{\mathrm{S} 59 \mathrm{~L}}$ proteins (i.e.,
RIPA-insoluble and urea-soluble) by immunoblotting (Fig. 4a). Co-expression of $\mathrm{CHCHD} 10^{W T}$ with $\mathrm{CHCHD} 10^{S 59 \mathrm{~L}}$ did not suppress the accumulation of insoluble CHCHD10 $559 \mathrm{~L}$ (Fig. 4a), which was consistent with the presence of punctate structures of CHCHD $10^{S 59 L}$ in elongated mitochondria by co-expressing CHCHD10 ${ }^{W T}$ (Fig. 3d, e). Therefore, CHCHD10 $559 \mathrm{~L}$ clearly forms insoluble aggregates in mitochondria. Again, $\mathrm{CHCHD} 10^{\mathrm{WT}}$ co-expression did not affect the insolubility and aggregate formation. However, it is still unclear whether the presence of insoluble $\mathrm{CHCHD} 10^{\mathrm{S} 59 \mathrm{~L}}$ is more relevant to toxicity than that of other intermediate species, such as oligomers.

CHCHD10 ${ }^{\text {S59L }}$ induces TDP-43 insolubility and mitochondrial translocation. Mutations in TARDBP cause ALS and FTD, and cytoplasmic TDP-43 aggregates are a hallmark in most patients with ALS and/or FTD ${ }^{37,38}$. In addition, mitochondrial translocation of TDP-43 and toxicity is reported in cell cultures and patient tissues ${ }^{25}$. Therefore, we hypothesized that CHCHD10 affects TDP-43 aggregation or mitochondrial translocation. We examined insoluble TDP-43 levels after CHCHD10WT and $\mathrm{CHCHD} 10^{S 59 L}$ transfection. Strikingly, $\mathrm{CHCHD} 10^{S 59 L}$ expression increased insoluble TDP-43, whereas $C H C H D 10^{W T}$ expression decreased insoluble TDP-43 (Fig. 4b). Co-expression of CHCHD10 ${ }^{W T}$ with $C H C H D 10^{S 59 L}$ suppressed CHCHD10 $559 L_{-}$ induced insoluble TDP-43 (Fig. 4b).

We next determined whether CHCHD10 ${ }^{W T}$ and CHCHD $10^{S 59 L}$ expression affects mitochondrial translocation of TDP-43. Despite similar expression levels in total lysates, exogenously expressed TARDBPWT and three pathogenic mutants (G298S, A315T, and A382T) showed an elevated amount in the mitochondrial fraction of $\mathrm{CHCHD} 10^{S 59 L_{-}}$expressing cells over that of empty vector-transfected cells. We observed decreased mitochondrial distribution of TDP-43WT and its mutants in $C H C H D 10^{W T}$-expressing cells (Fig. 4c, d). Furthermore, co-expression of CHCHD10WT with $\mathrm{CHCHD} 10^{S 59 L}$ reduced the amount of TDP-43 and its mutants in the mitochondrial fraction, which was increased by $\mathrm{CHCHD} 10^{S 59 L}$ expression (Fig. 4e, f). Immunofluorescence staining confirmed the increased mitochondrial localization of TDP-43 in

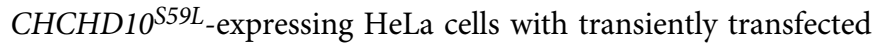


a

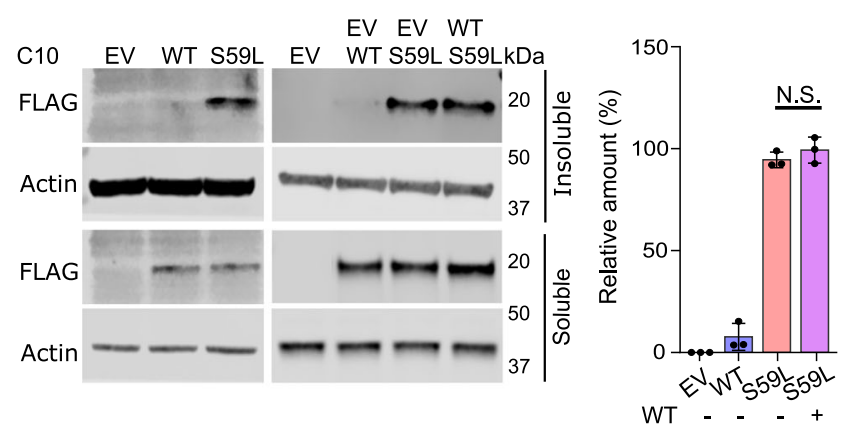

b

C10 WT EV EV EV +
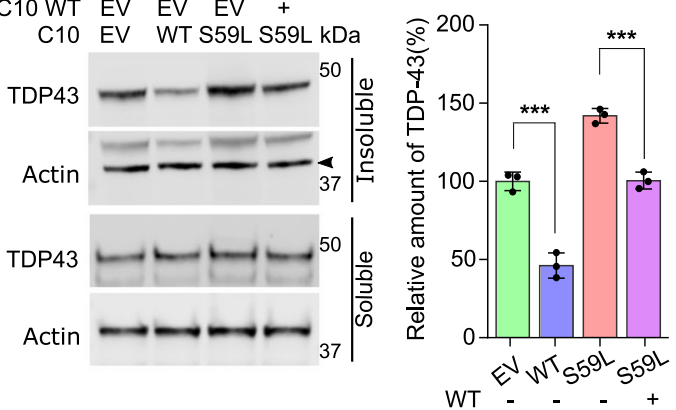

C
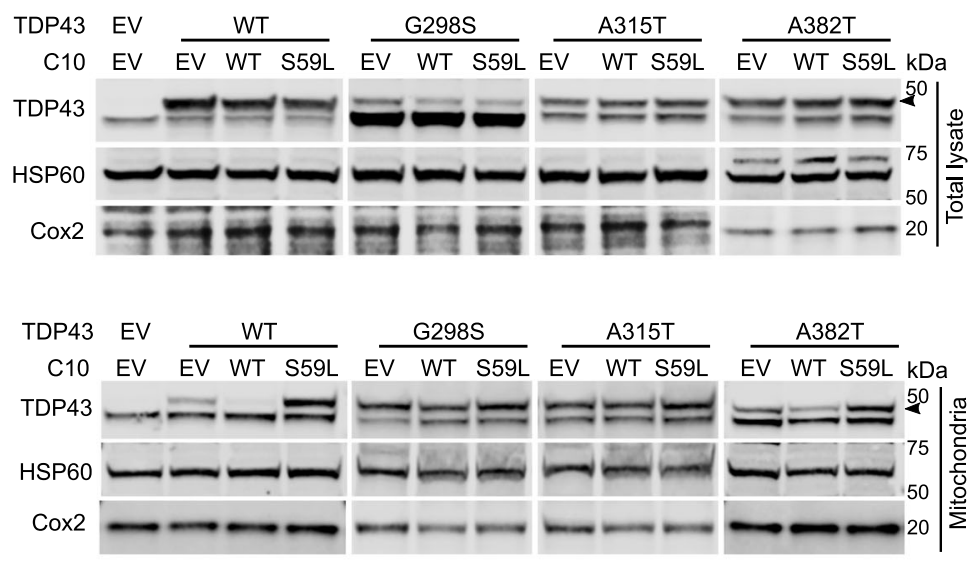

e
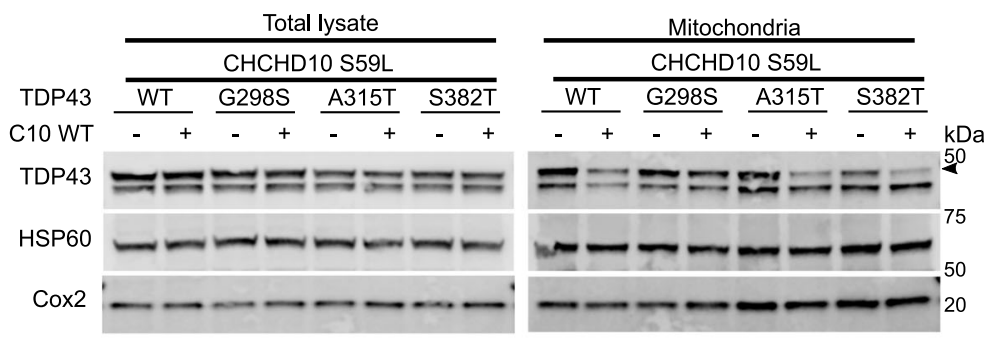

g

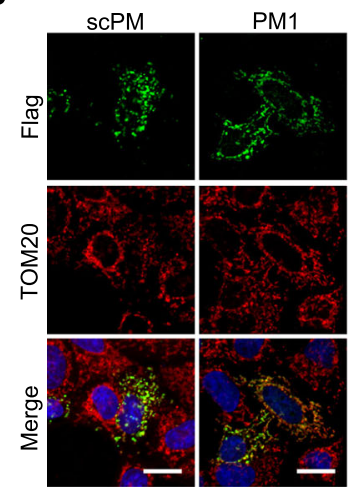

h

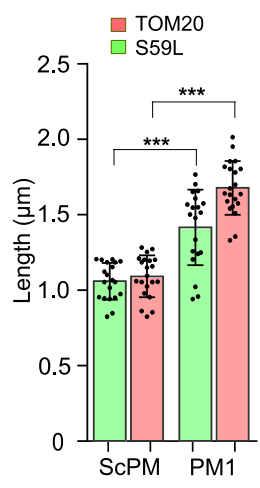

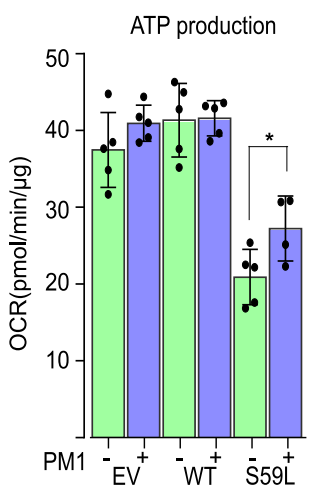

d

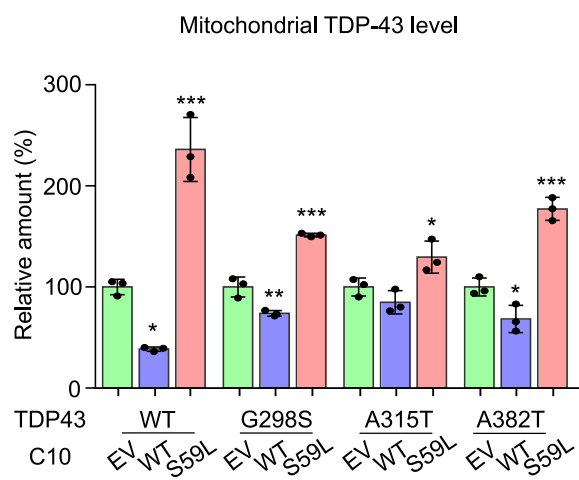

f

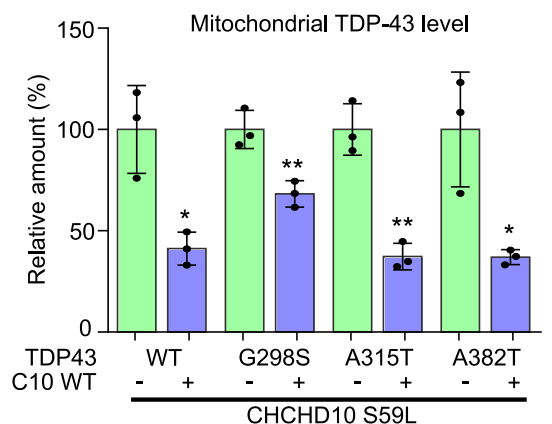

i

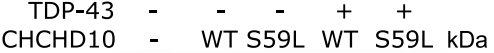

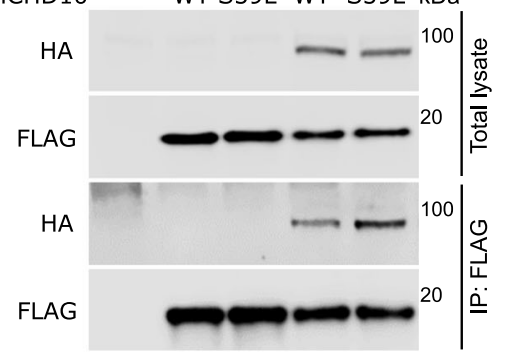

TDP-43 (Supplementary Fig. 4b). In addition, increased cytoplasmic and mitochondrial localization of endogenous TDP-43 was observed in SH-SY5Y neuroblastoma cells expressing CHCHD10 ${ }^{S 59 L}$ (Supplementary Fig. 4c, d). An in vivo experiment with a humanized Drosophila model replacing the entire TBPH coding region with human $T A R D B P^{39}$ revealed significantly increased TDP-43 association with mitochondria in $\mathrm{C}_{2} \mathrm{C}_{10 \mathrm{H}^{S 81 L}}$ expressing muscle tissues, as compared to $C 2 \mathrm{C}_{10 H^{W T}}$-expressing muscles (Supplementary Fig. 4e). These observations suggest that the prevention of TDP-43 mitochondrial translocation, as demonstrated by Wang et al. ${ }^{25}$, may be a potential therapeutic strategy for CHCHD10-induced ALS-FTD. To test whether 
Fig. 4 CHCHD10 $559 L$ increases TDP-43 insolubility and mitochondrial translocation. a HeLa cells were transfected with FLAG-tagged CHCHD10WT or $\mathrm{CHCHD10} 559 \mathrm{~L}$. After $24 \mathrm{~h}$, cells were subjected to sequential protein extraction with RIPA and urea buffers. Immunoblotting was conducted with anti-FLAG and anti-actin (loading control) antibodies. Data are shown as mean \pm SD (one-way ANOVA and post hoc Tukey's test, two-sided, NS, not significant; $n=$

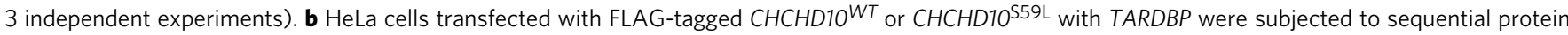
extraction with RIPA and urea buffers. Immunoblotting was performed with anti-TDP-43 or anti-actin (loading control) antibodies. Data are shown as mean \pm SD (one-way ANOVA and post hoc Tukey's test, two-sided, ${ }^{\star \star *} p=0.000738$ for $W T,{ }^{* \star *} p=0.000533$ for S59L + WT; $n=3$ independent experiments). c, d HeLa cells were co-transfected with FLAG-tagged TARDBPWT or disease-causing mutants combined with empty vector (EV), CHCHD10WT, or $\mathrm{CHCHD10} 559 \mathrm{~L}$. After $24 \mathrm{~h}$, mitochondria were fractionated. Immunoblotting was conducted with anti-TDP-43 (arrowhead indicates transfected TDP-43), anti-HSP60, and anti-TOM2O (loading controls) antibodies. Data are shown are mean \pm SD (one-way ANOVA and post hoc Dunnett test, two-sided, ${ }^{\star} p=0.01284$ and ${ }^{\star \star \star} p=0.00021$ in TDP-43 WT, ${ }^{\star \star} p=0.0032$ and ${ }^{\star \star \star} p=7.9 \mathrm{e}-05$ in G298S, ${ }^{\star} p=0.0475$ in A315T, ${ }^{\star} p=0.02604$ and ${ }^{\star \star \star} p=0.00031$ in A382T). e, f HeLa cells were co-transfected with FLAG-tagged TARDBPWT or disease-causing mutants combined with EV or CHCHD10WT. After $24 \mathrm{~h}$, mitochondria were fractionated. Immunoblotting was conducted with anti-TDP-43 (arrowhead indicates transfected TDP-43) or anti-HSP60 and anti-TOM20 (loading control) antibodies. Data are shown as mean \pm SD (two-sided $t$ test, ${ }^{\star} p=0.011759,{ }^{\star \star} p=0.008552,{ }^{\star} p=0.001604$, and ${ }^{* \star} p=0.018798$ for TDP-43 WT, G298S, A315T, and A382T, respectively). $\mathbf{g}$ HeLa cells expressing CHCHD10 ${ }^{559 L}$ were treated with a control peptide $(\mathrm{scPM}, 5 \mu \mathrm{M})$ or TDP-43 inhibitor (PM1, $5 \mu \mathrm{M}$ ). Representative images of HeLa cells stained with anti-FLAG (green, CHCHD10559L) and TOM20 (red, mitochondria) antibodies. Data are shown as mean \pm SD (two-sided $t$ test, ${ }^{\star \star \star} p=1.26 \mathrm{e}-06$ and $8.98 \mathrm{e}-15$ for S59L and Tom 20 , respectively; $n=20$ cells from three independent experiments). Scale bar $=20 \mu \mathrm{m}$. $\mathbf{h}$ HeLa cells expressing $C H C H D 10^{S 59 L}$ were treated with a control peptide (scPM, $2 \mu \mathrm{M}$ ) or TDP43 inhibitor (PM1, $2 \mu \mathrm{M}$ ). Mitochondrial respiration was measured by Seahorse XF Cell Mito Stress tests. A representative graph of a single experiment is shown (mean $\pm \mathrm{SD}$ ). Actual statistical analyses were performed with four independent experiments (one-way ANOVA and post hoc Dunnett test, twosided, comparison with $\mathrm{EV},{ }^{\star} p=0.024685$; Detailed information on statistical analyses is available in Supplementary Fig. $9 \mathrm{f}$ ). $\mathbf{i} \mathrm{HEK} 293$ cells were transfected with FLAG-tagged $\mathrm{CHCHD10}$ WT or $\mathrm{CHCHD10^{559L }}$ with/without HA-tomato-tagged TARDBP. After $24 \mathrm{~h}$, lysates were subjected to coimmunoprecipitation with anti-FLAG (M2) affinity gels. Immunoblotting was conducted with anti-FLAG (CHCHD10) and HA (TDP-43) antibodies. A representative image from three independent experiments is shown.

inhibition of TDP-43 mitochondrial translocation recovers CHCHD $10^{S 59 L}$-induced mitochondrial morphologic and functional defects, we treated $C H C H D 10^{S 59 L}$-transfected cells with the PM1 peptide inhibitor of TDP-43 mitochondrial translocation ${ }^{25}$. Notably, the morphologic and functional defects caused by $\mathrm{CHCHD} 10^{S 59 L}$ were ameliorated by PM1 (Fig. $4 \mathrm{~g}, \mathrm{~h}$ and Supplementary Fig. 4f), suggesting that increased mitochondrial translocation are critical to $\mathrm{CHCHD} 10^{S 59 L}$-induced cell toxicity. Although it has been demonstrated that TDP-43 can translocate into mitochondria in diseases ${ }^{25}$ or stressed conditions ${ }^{40}$, it is not clear how TDP-43 can be abnormally retained in mitochondria upon $C H C H D 10$ mutation. We hypothesized that $C H C H D 10^{S 59 L}$ increases the binding capacity of CHCHD10 to TDP-43 in mitochondria because CHCHD10 lacks catalytic activity, and physical interactions between CHCHD10 and TDP-43 are reported $^{19}$. To test this hypothesis, HEK293T cells were transfected with $C H C H D 10^{W T}-$ FLAG or CHCHD10 $559 L_{-}$FLAG with/without TDP-43-tomato-HA, followed by immunoprecipitation with anti-FLAG affinity beads. Significantly, $\mathrm{CHCHD} 10^{559 \mathrm{~L}}$ showed an increased binding capacity to TDP43 (Fig. $4 \mathrm{i}$ and Supplementary Fig. 4g). This was reaffirmed by using another combination of purification tags: CHCHD10-HA and TDP-43-FLAG (Supplementary Fig. 4h).

PINK1/parkin mediates dominant degeneration in the $\mathrm{C2C10H}^{\text {S81L }}$ Drosophila model. Because $C 2 \mathrm{C} 10 \mathrm{H}^{S 81 L}$ and CHCHD $10^{S 59 L}$ expression caused consistent mitochondrial toxicity in Drosophila and human cells, respectively, we have further dissected genetic pathways of $C 2 C 10 H^{S 81 L}$-mediated cell toxicity using our Drosophila model. Because of notable mitochondrial fragmentation and functional respiratory defects, we hypothesized that the genes involved in mitochondrial dynamics or mitochondrial quality control are effectors of $\mathrm{CHCHD} 10^{S 59 L_{-}}$ driven mitochondrial pathogenesis. We performed genetic interaction studies using the Drosophila $\mathrm{C} 2 \mathrm{C} \mathrm{OH}^{S 81 L}$ eye model with various RNAi, classical deficiency, and duplication lines (Supplementary Table 1). The most potent dominant suppressor was PINK1. Downregulation of PINK1 by RNAi recovered the rough eye phenotype produced by two copies of $\mathrm{C} 2 \mathrm{C} 10 \mathrm{H}^{S 81 L}$, whereas PINK1 overexpression enhanced the rough eye phenotype (Fig. 5a). Moreover, RNAi-mediated depletion of park (i.e., the Drosophila gene encoding parkin), a downstream partner of PINK1 for the mitochondrial quality control, also marginally rescued the rough eye phenotype (Fig. 5a).

We also tested several other genes that are parallel or downstream of the PINK1/parkin pathway, such as mul1, ari1, march5, Drp1, marf, and TER94 (Supplementary Table 1). Only overexpression of marf, a Drosophila ortholog of MFN (i.e., mitofusin), showed mild suppressive effects with consistent RNAi-mediated enhancement (Fig. 5a). This indicates that Marf may be a downstream effector of the PINK1/parkin pathway during $\mathrm{CHCHD} 10^{S 59 L}$-mediated pathogenesis. However, this observation does not exclude the other genes tested from playing a role in $\mathrm{C} 2 \mathrm{C}_{10 \mathrm{H}^{S 81 L}}$-mediated pathogenesis. Consistent with our findings in the eye model, RNAi-mediated depletion of PINK1 also recovered $\mathrm{C} 2 \mathrm{C}_{10 H^{S 81 L}}$-dependent indirect flight muscle degeneration (Fig. 5b). PINK1 knockdown rescued sarcomere structure, extended mitochondrial length, and increased ATP production (Fig. 5b, c), improving $\mathrm{C} 2 \mathrm{C}_{10} \mathrm{H}^{S 81 L}$-induced defects, including abnormal wing posture and flight ability (Supplementary Fig. 5a). Human CHCHD10 $559 L$-induced rough eye phenotypes were also rescued by PINK1 knockdown. (Supplementary Fig. 5b). These results suggest that $\mathrm{C} 2 \mathrm{C} 10 \mathrm{H}^{\mathrm{S} 81 \mathrm{~L}}$ (and human $\mathrm{CHCHD} 10^{\mathrm{S} 59 \mathrm{~L}}$ ) activates the PINK1/parkin pathway to generate dominant cell toxicity in the Drosophila system.

PINK1/parkin mediates dominant cell toxicity in HeLa cells and patient-derived fibroblasts. RNAi-mediated knockdown of PINK1 remarkably rescued $C H C H D 10^{S 59 L}$-induced mitochondrial morphologic and functional defects in HeLa cells (Fig. $5 \mathrm{~d}-\mathrm{f}$ and Supplementary Fig. 5c). Furthermore, the expression of CHCHD10 $559 L$ in PINK1-knockout HeLa cells did not cause mitochondrial fragmentation (Supplementary Fig. 5d). Although MFN2 knockdown marginally affected the CHCHD10 $559 \mathrm{~L}_{-}$ induced mitochondrial defects in HeLa cells (Fig. 5d-f), the simultaneous knockdown of the two paralogs MFN1 and MFN2 enhanced the mitochondrial defects (Supplementary Fig. 5e, f). However, overexpression of MFN2-YFP was uninterpretable because of its strong mitochondrial clustering activity ${ }^{41}$, although we detected marginal beneficial effects on respiratory function 
a

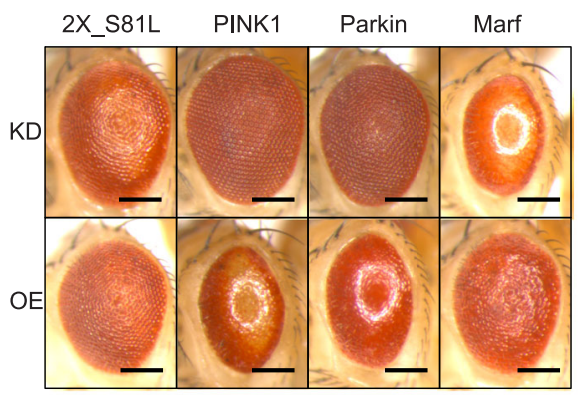

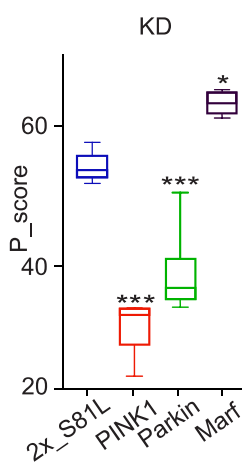

KD

b

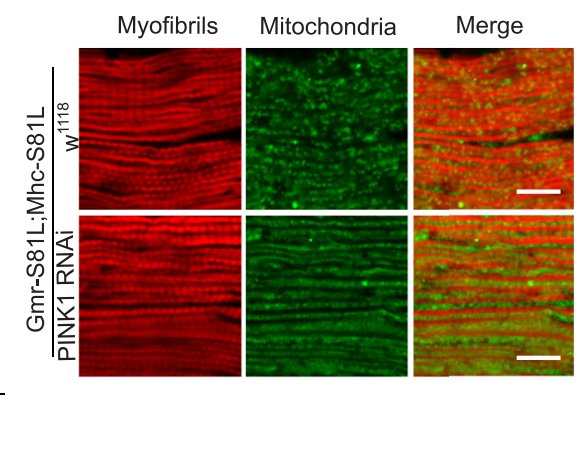

C

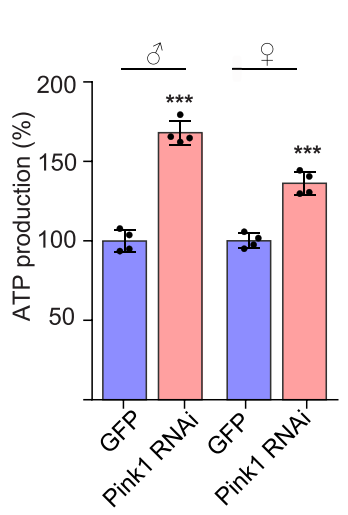

d

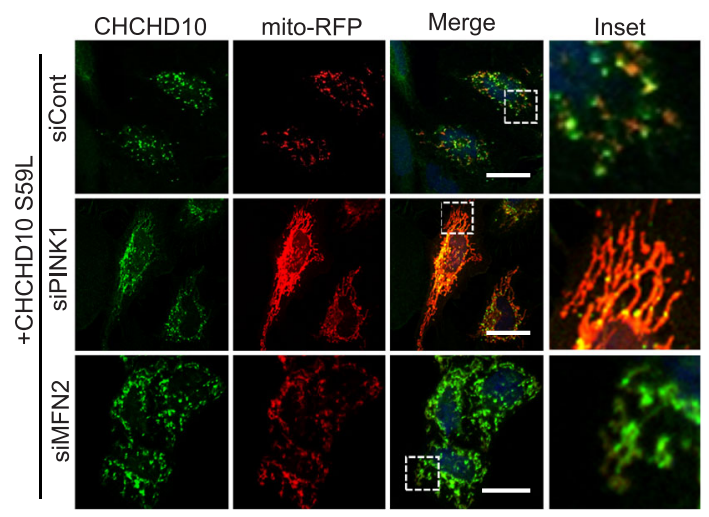

e

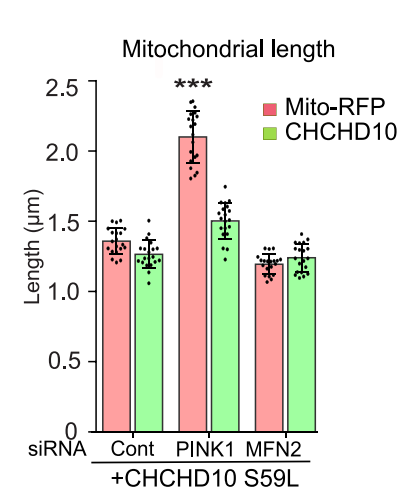

f
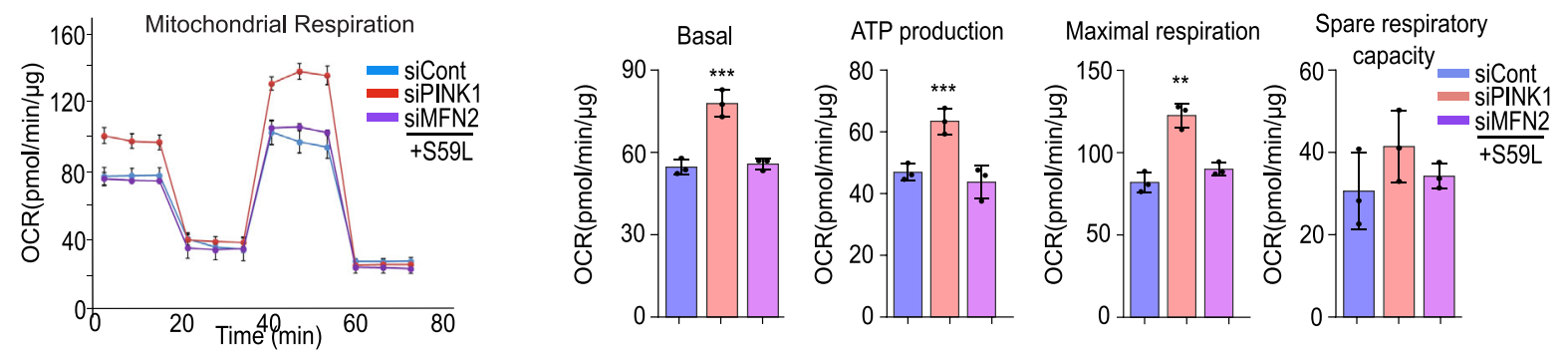

g

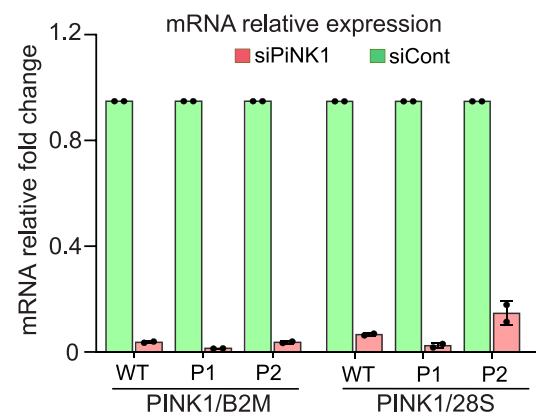

h
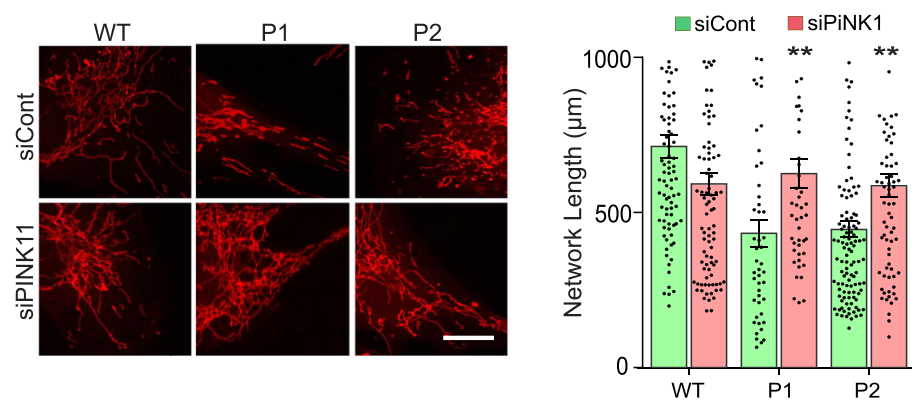

(Supplementary Fig. 5g, h). In our Drosophila model, both Drp1 knockdown and overexpression exhibited detrimental effects (Supplemental Table 1). However, RNAi-mediated depletion of DRP1 in HeLa cells successfully reversed $C H C H D 10^{S 59 L}$-induced mitochondrial fragmentation and exerted beneficial effects on respiratory function (Supplementary Fig. 5i, j). Consistently, CHCHD10 ${ }^{S 59 L}$-mediated respiratory defects were slightly enhanced by DRP1-YFP co-expression, although it was not statistically significant. (Supplementary Fig. 5h). Indeed, CHCHD $10^{S 59 \mathrm{~L}}$ aggregates were nearly absent with $D R P 1$ knockdown (Supplementary Fig. 5g, i), in contrast with persistent aggregates with PINK1 knockdown (Fig. 5d, e). We speculated that pre-elongated mitochondria with DRP1 knockdown reduces the local concentration of $\mathrm{CHCHD} 10^{\mathrm{S} 59 \mathrm{~L}}$ proteins and thus prevents aggregate formation. Although it is apparent that modulation of mitochondrial dynamics may be beneficial in reducing 
Fig. 5 CHCHD10 559 -induced degeneration is rescued by Pink1 downregulation. a RNAi-mediated knockdown effects of PINK1, Park, and their downstream target, Marf (Drosophila mitofusin) on $\mathrm{C}_{2} \mathrm{C}_{10 H} \mathrm{~S}^{51 \mathrm{~L}}$-induced eye phenotypes. Boxes indicate median and 25th and 75th percentiles (one-way ANOVA and post hoc Dunnett test, two-sided, or Fisher's least significant difference, ${ }^{\star \star \star} p=1.3 e-06,0.0004$ and ${ }^{\star} p=0.0486$ in $\mathrm{KD},{ }^{\star} p=0.04797,{ }^{\star \star \star} p=0.00015$, and ${ }^{*} p=0.0588$ in OE for PINK1, parkin and Marf, respectively; $n=4-11$ flies from a single experiment; results were verified with multiple fly lines; see Supplementary Table 1). Scale bar $=200 \mu \mathrm{m}$. b RNAi-mediated knockdown of PINK1 in muscles rescued C2C10H ${ }^{S 81 L}$-induced muscle degeneration and mitochondrial defects. Representative images from three independent experiments. Scale bar $=20 \mu \mathrm{m}$. c ATP levels in thoraxes from 10-day-old flies were measured. Data are shown as mean \pm SD (two-sided $t$ test, ${ }^{\star \star \star} p=1.22 \mathrm{e}-05$ for male and 0.000157 for female; $n=4$ independent experiments). $\mathbf{d}$, e HeLa cells were transfected with siRNAs targeting PINK1 or MFN2. After $18 \mathrm{~h}$, the cells were transfected with CHCHD10 $559 \mathrm{~L}$ and Mito-RFP. Representative images of transfected HeLa cells immunostained with antibodies against FLAG. Scale bar $=20 \mu \mathrm{m}$. Quantification of Mito-RFP and CHCHD10S59L-FLAG signal length. Data are shown as mean \pm SD (one-way ANOVA and post hoc Dunnett test, two-sided, comparison with $E V ;{ }^{* * *} p<2 e-16 ; n=20$ cells from three

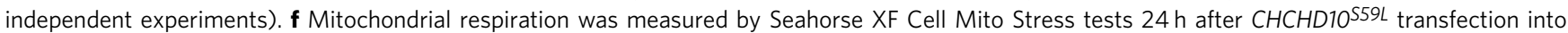
siRNA-treated cells. Graphs of a single representative experiment are shown (mean \pm SD). Actual statistical analyses were performed with 8 independent experiments (one-way ANOVA and post hoc Dunnett test, two-sided, comparison with EV, ${ }^{\star \star \star} p=0.00034,0.00031$ and ${ }^{\star \star} p=0.0055$ for basal, ATP and maximal level, respectively; detailed information on statistical analyses is available in Supplementary Fig. 9g). $\mathbf{g}$ RT-qPCR analysis of PINK1 in siRNAtransfected fibroblasts. Results are shown as fold change of PINK1 mRNA expression in siPINK1 transfected fibroblasts relative to siControl transfected fibroblasts. Data were normalized to $\beta-2$ macroglobulin (B2M) or 28S. Results shown are mean \pm SEM from two independent experiments. $\mathbf{h}$ PINK1 downregulation by siRNA treatment reversed mitochondrial network fragmentation in $\mathrm{CHCHD10} 559 \mathrm{~L} /+$ patient fibroblasts. Patients (P1, P2) or wild-type (WT) fibroblasts were transfected with control siRNA (siCont) or PINK1 siRNA (siPINK1). P1 and P2 correspond to patient V-10 ${ }^{1}$ and to patient IV-3 ${ }^{11}$, respectively. Representative images of the mitochondrial network with MitoTracker staining. Scale bar $=20 \mu \mathrm{m}$. Mitochondrial network length was quantified from two independent experiments with 48-116 randomly selected individual cells. Differences between siCont and siPINK1 were analyzed by two-sided Mann-Whitney test $\left({ }^{* \star} p=0.0024\right.$ and 0.0013 for patients 1 and 2 , respectively).

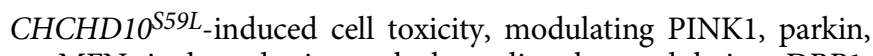
or MFN is less detrimental than directly modulating DRP1. Finally, we examined whether RNAi-mediated downregulation of PINK1 affects the fragmented mitochondrial network observed in fibroblasts derived from patients carrying a CHCHD $10^{S 59 L}$ allele ${ }^{1,11}$. Transfection with PINK1 small interfering RNA (siRNA) successfully reduced the amount of PINK1 transcripts in both control and patient fibroblasts (Fig. 5g). MitoTracker staining and analysis for control and PINK1 siRNA-transfected cells showed that the loss of PINK1 rescued the fragmented mitochondrial network found in patient-derived fibroblasts (Fig. 5h).

Parkin-mediated mitophagy induces cell toxicity. Upon mitochondrial stress or damage, PINK1 accumulates in mitochondria ${ }^{42}$ and recruits parkin by phosphorylating ubiquitin and other substrates, including parkin, resulting in MFN1/2 degradation and mitophagy to remove damaged mitochondria ${ }^{43-46}$. The suppressive effects of PINK1 and PRKN knockdown suggest that CHCHD10 ${ }^{\text {S59L }}$ induces PINK1 accumulation in mitochondria, activating the PINK1/parkin pathway. Indeed, PINK1-YFP accumulated in the mitochondria of

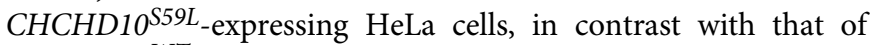
CHCHD10 ${ }^{W T}$ overexpression (Fig. 6a, c). Because PRKN expression is deficient in HeLa cells, stable cell lines expressing YFP-Parkin (HeLa YFP-Parkin) have been established and widely used to study parkin-mediated mitophagy ${ }^{42}$. When CHCHD $10^{S 59 L}$ was transiently expressed in HeLaYFP-Parkin cells, YFP-Parkin was also recruited to mitochondria (Fig. 6b, d), clearly demonstrating that $\mathrm{CHCHD} 10^{\mathrm{S} 59 \mathrm{~L}}$ induces PINK1 stabilization, accumulation on mitochondria, and subsequent parkin recruitment. This suggests that PINK1/parkinmediated mitophagy is toxic in this system. Indeed, enhancing autophagy by Atg1 expression in the $C 2 \mathrm{C}_{10 H} \mathrm{H}^{S 81 L}$ eye model was synergistically lethal (Supplementary Table 1), although beneficial effects of Atg1 overexpression have been reported in other Drosophila disease models ${ }^{47,48}$. However, strong mitochondrial abnormalities in PRKN-deficient HeLa cells also indicate the presence of parkin-independent toxic mechanisms. To explain $\mathrm{CHCHD} 10^{559 \mathrm{~L}}$-induced cell toxicity in both PRKN-deficient HeLa cells and HeLaYFP-Parkin, we hypothesized that PINK1mediated, parkin-independent mitophagy ${ }^{49}$ is highly activated in PRKN-deficient HeLa cells by CHCHD10 $0^{\text {S5 }}$ and that dysregulated mitophagy is amplified by Parkin in HeLaYFP-Parkin. To test this hypothesis, we first assessed LC3 conversion and accumulation in mitochondria to examine whether autophagosome formation is activated by $\mathrm{CHCHD} 10^{559 \mathrm{~L}}$ expression in HeLa cells.

In both $\mathrm{HeLa}$ and $\mathrm{HeLa}$ YFP-Parkin cells, CHCHD10 $559 \mathrm{~L}$ expression increased LC3 accumulation (Fig. 6e, f) and LC3 conversion (Fig. 6g). However, only small portions of LC3 accumulation co-localized with mitochondria in $P R K N$-deficient HeLa cells, as compared with strong mitochondrial colocalization of LC3 accumulation in HeLaYFP-Parkin cells (Fig. 6e, h). Co-staining of lysosomes and mitochondria also revealed limited lysosomal marker staining in the mitochondria of $P R K N$ deficient Hela cells (Supplementary Fig. 6a). To confirm this observation, we determined the mitochondrial LC3 levels after fractionating mitochondria. Although LC3-II amount increased in mitochondrial fractions of $\mathrm{CHCHD} 10^{S 59 L}$-transfected HeLa and HeLaYFP-Parkin, significantly more LC3-II were detected in HeLaYFP-Parkin as expected (Supplementary Fig. 6b). A mitochondrial fraction from $C H C H D 10^{S 59 L}$ - transfected SH-SY5Y expressing parkin endogenously showed clear LC3-II accumulation (Supplementary Fig. 6c). To determine whether the increased LC3 levels from $C H C H D 10^{S 59 L}$-transfected cells correspond with mitophagic turnover, we examined mitolysosomes using the mito-QC system in HeLa, HeLa YFP-Parkin, and SH-SY5Y cells. Consistent with LC3 conversion and accumulation, mito-QCpositive mitolysosomes (GFP-negative/mCherry-positive) were

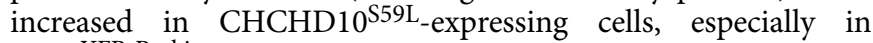
HeLa YFP-Parkin and SH-SY5Y (Fig. 6i, j and Supplementary Fig. $6 \mathrm{~d}, \mathrm{e})$. Therefore, it is apparent that mitophagy induction is a major phenomenon when $\mathrm{CHCHD} 10^{\mathrm{S} 59 \mathrm{~L}}$ is expressed. However, it is still unclear whether PINK1-mediated, parkin-independent mitophagy mildly induced by $\mathrm{CHCHD} 10^{\mathrm{S} 59 \mathrm{~L}}$ contributes to the cell toxicity in PRKN-deficient HeLa cells. Thus, to test whether the induction of PINK1-mediated, parkin-independent mitophagy by $\mathrm{CHCHD} 10^{\mathrm{S} 59 \mathrm{~L}}$ is critical for generating cell toxicity in $P R K N$-deficient HeLa cells, we reduced the expression of two mitophagy receptors involved in PINK1-mediated, parkinindependent mitophagy, NDP52 and optineurin $(O P T N)^{49}$ by RNAi. Knockdown of these two receptors had no effect on CHCHD $10^{S 59 L}$-mediated cell toxicity in PRKN-deficient HeLa cells, but increased respiratory activity significantly in HeLa YFP- 
a

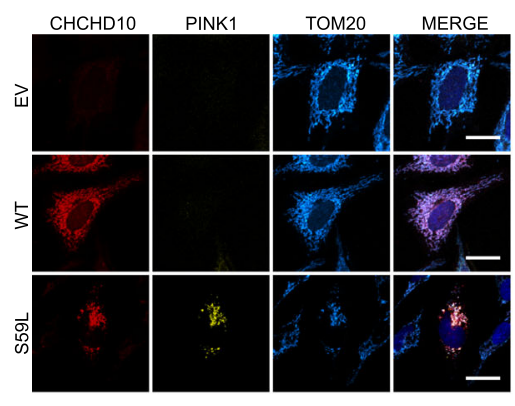

e

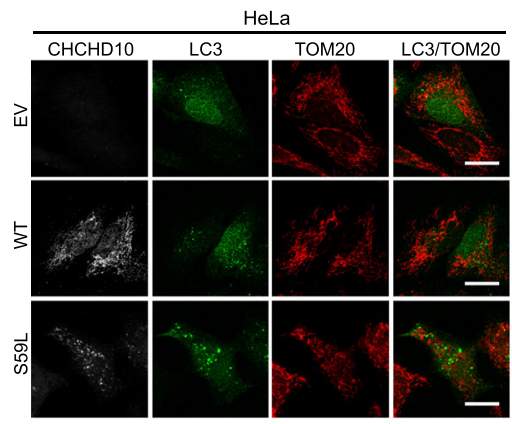

b

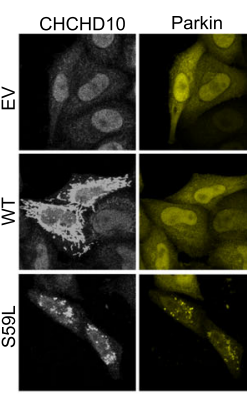

C

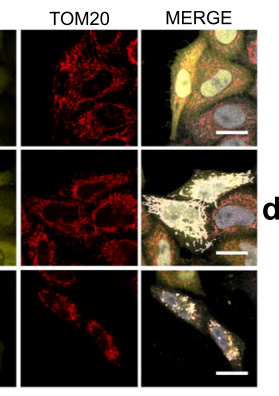

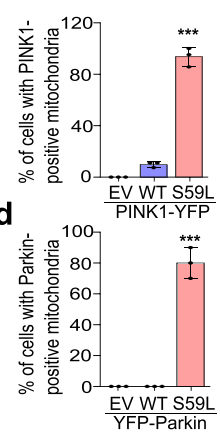

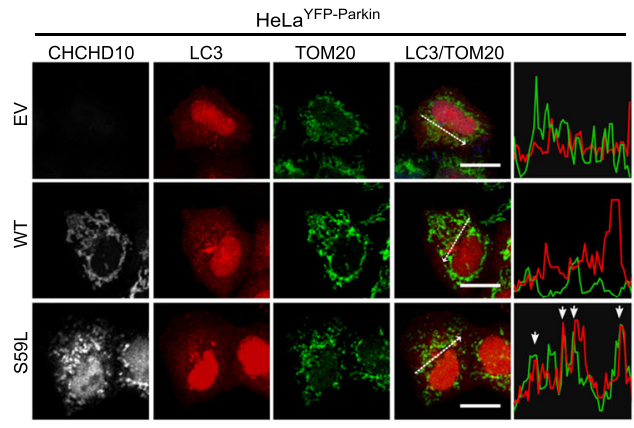

h

f

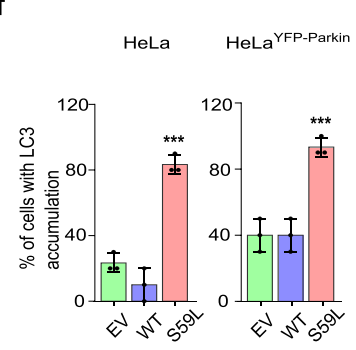

i

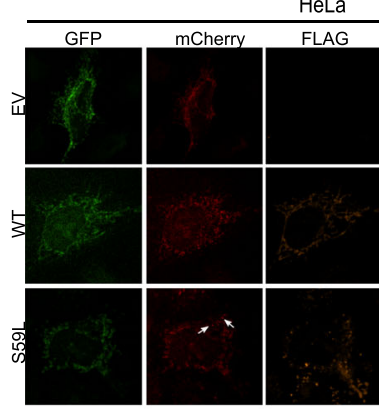

$\mathbf{k}$

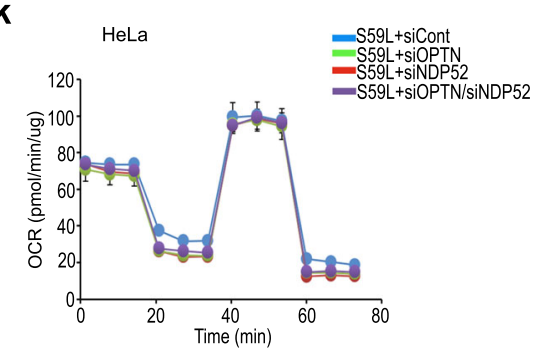

g

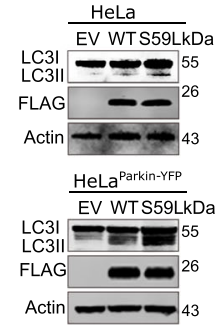

Actin $\longrightarrow$ - 43

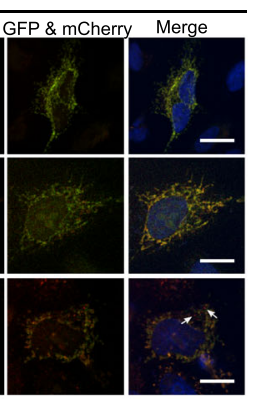

I
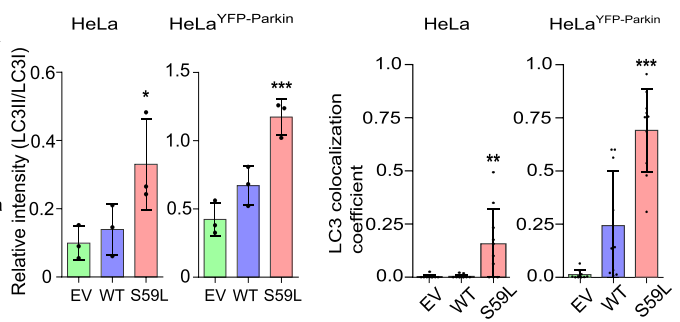

j

jeLa ${ }^{\text {YFP-Parkin }}$
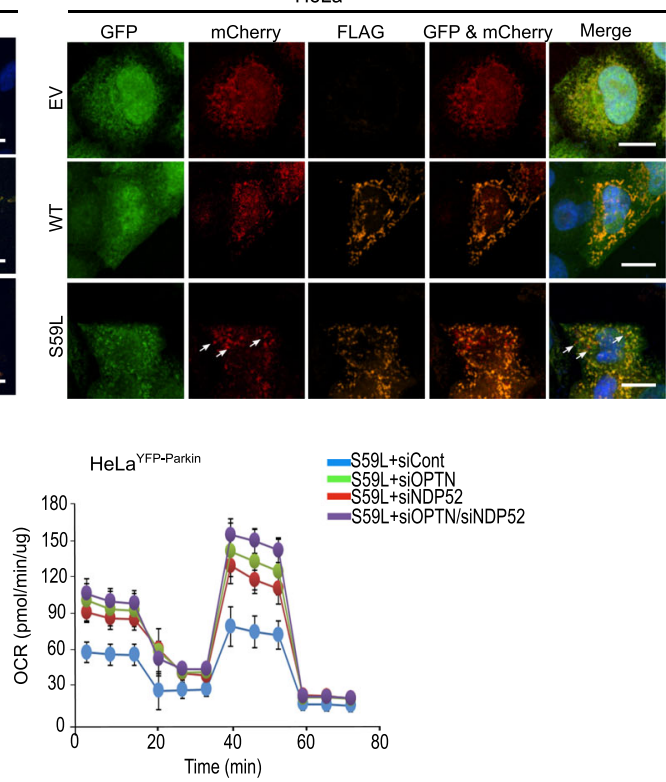

Parkin cells (Fig. 6k, 1 and Supplementary Fig. 6f-h). Therefore, the $\mathrm{PINK} 1 /$ parkin-mediated mitophagy pathway is one of the major toxicogenic pathways when Parkin exists. However, without Parkin, the mitophagic pathway is not essential to generate the substantial cell toxicity observed in PRKN-deficient HeLa cells,

indicating that PINK1 downstream factors other than Parkin can also mediate the cell toxicity independent of Parkin.

Modulating PINK1 downstream pathways mitigates

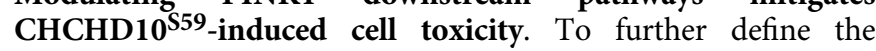


Fig. 6 CHCHD10 559 -induced toxicity is mediated by mitophagy when Parkin exists. a HeLa cells and b HeLaYFP-Parkin cells were transfected with FLAGtagged CHCHD10 S59L and PINK1-YFP. The transfected HeLa cells were immunostained with antibodies against FLAG and TOM20 $24 \mathrm{~h}$ after transfection. Scale bar $=20 \mu \mathrm{m}$. c, d Percentage of HeLa cells showing PINK1-YFP (c) and YFP-Parkin (d) accumulation in mitochondria; $n=3$ independent experiments, $\geq 50$ cells for each group. Data are shown as mean \pm SD (one-way ANOVA and post hoc Dunnett test, two-sided, comparison with empty vector [EV]; ${ }^{* \star \star} p$ $=3.7 \mathrm{e}-07$ (c) and $4.9 \mathrm{e}-06$ (d). e HeLa cells and HeLa YFP-Parkin cells were co-transfected with FLAG-tagged CHCHD10 $559 \mathrm{~L}$ and GFP-LC3 or mCherry-LC3, respectively. Representative images of transfected cells immunostained with antibodies against FLAG and TOM20. Graphs show the fluorescence intensity profiles of mCherry-LC3 (red), and TOM2O (green) along the region marked by an arrow in HeLaparkin-YFP merged image. Arrowheads indicate highly mCherry-LC3 and Tom20 merged region. Scale bar $=20 \mu \mathrm{m}$. f Percentage of cells showing GFP- or mCherry-LC3 accumulation. Data are shown as mean \pm SD (one-way ANOVA and post hoc Dunnett test, two-sided, ${ }^{\star \star \star} p=0.00011$ for HeLa and 0.00056 in HeLaYFP-parkin; $n=3$ independent experiments, with $\geq 20$ cells for each group). $\mathbf{g}$ Immunoblot of LC3 and CHCHD10 $559 \mathrm{~L}$ expression. Data are shown as mean \pm SD (one-way ANOVA and post hoc Dunnett test, two-sided, ${ }^{*} p=0.0387,{ }^{\star \star \star} p=0.00084 ; n=3$ independent experiments). h GFP-LC3 and mCherry-LC3 co-localization with TOM20 in HeLa cells and HeLaYFP-Parkin cells. Co-localization was measured by Pearson's correlation coefficient. Data are shown as mean \pm SD (one-way ANOVA and post hoc Dunnett test, two-sided, ${ }^{\star \star} p=0.0022,{ }^{\star \star \star} p=1.8 \mathrm{e}-08 ; n=10$ cells from three independent experiments). $\mathbf{i}$ HeLa cells and $\mathbf{j}$ HeLaYFP-Parkin cells were cotransfected with FLAG-tagged CHCHD10WT or $\mathrm{CHCHD10} 559 \mathrm{~L}$ and mito-QC. Representative images of transfected cells (three independent experiments) immunostained with anti-FLAG antibody. Arrows indicate mitolysosomes (GFP-negative/mCherry-positive). Scale bar $=20 \mu \mathrm{m}$. $\mathbf{k}$ HeLa and I HeLaYFPParkin cells were transfected with siRNAs targeting NDP52 or/and OPTN. At $24 \mathrm{~h}$ after siRNA transfection, mitochondrial respiration was measured by Seahorse XF Cell Mito Stress tests. Representative graphs of a single experiment are shown (mean \pm SD). Statistical analyses were performed with three independent experiments (see Supplementary Fig. 6g, h).

parkin-independent toxic pathway of PINK1, we tested whether the known downstream components ND42, sicily, Miro, and mitofilin modulate the $C 2 \mathrm{C}_{10} \mathrm{H}^{S 81 L}$-induced rough eye phenotype (Supplementary Table 1). Only co-expression of PINK1 phosphorylation-null Mitofilin ${ }^{50}$ showed mild but obvious rescue

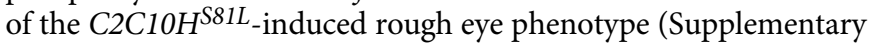
Fig. 7a), whereas RNAi-mediated knockdown of Mitofilin enhanced this phenotype (Supplementary Fig. 7b). Similarly, coexpression of PINK1 phosphorylation-null MFN2 $2^{S 378} 51$ with CHCHD $10^{S 59 L}$ in HeLa cells reduced the abnormal mitochondrial phenotype (Fig. 7a and Supplementary Fig. 7c). These data suggest that fine modulation of the downstream events caused by PINK1-mediated phosphorylation can be an effective therapeutic strategy. Indeed, recently developed MFN2 agonists reversing mitochondrial defects in a Charcot-MarieTooth disease type $2 \mathrm{~A}$ model ${ }^{51}$ also rescued CHCHD10 $0^{559 L_{-}}$ induced morphologic and functional mitochondrial defects (Fig. 7b, c and Supplementary Fig. 7d, e). We also observed the moderately elongated mitochondrial phenotype in the indirect flight muscles of $\mathrm{C} 2 \mathrm{C}_{10 H} \mathrm{H}^{S 1 L}$-expressing flies reared on two MFN2 agonists (Fig. 7d), which also increased ATP production in flies expressing $\mathrm{C}^{2} \mathrm{C}_{10 H} \mathrm{H}^{S 81 L}$ (Fig. 7e) and in C9orf72-expressing flies with expanded GGGGCC repeats (Supplementary Fig. $7 f)^{52}$.

Inhibition of PINK1 activity mitigates $\mathrm{CHCHD10} 0^{\mathrm{S59}}$-induced cell toxicity. We hypothesized that inhibition of PINK1 kinase activity is a more effective and robust therapeutic strategy than regulating PINK1 expression level or its downstream substrates. Because of a lack of small-molecule inhibitors of PINK1, we used LALIGN to generate peptide sequences that may act as pseudo-

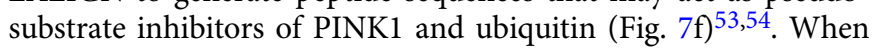
we treated HeLa cells with two putative peptide inhibitors of PINK1 via a protein delivery reagent, we observed that CHCHD10 $159 \mathrm{~L}$-mediated mitochondrial fragmentation was completely abolished in both pretreated and post-treated experiments (Fig. $7 \mathrm{~g}$ and Supplementary Fig. $7 \mathrm{~g}$, h). Immunoblotting and immunostaining with an anti-phospho-ubiquitin antibody confirmed that ubiquitin phosphorylation was reduced by peptide treatment (Fig. 7h, i). Peptide treatment also reduced PINK1 accumulation, along with decreased phospho-ubiquitin staining (Fig. 7i), suggesting that a positive feedback mechanism amplifies CHCHD10 $0^{S 59}$-mediated cell toxicity via PINK1 accumulation (Fig. 7j).
PINK1 knockdown does not affect TDP-43, but CHCHD10 WT prevents PINK1 accumulation. To determine whether the two axes of $C H C H D 10^{S 59 L}$-induced cell toxicity exhibit crosstalk, we examined TDP-43 insolubility and mitochondrial localization after RNAi-mediated PINK1 knockdown in CHCHD10 $0^{S 59 L}$ transfected HeLa cells. Although PINK1 knockdown rescued the morphologic and functional defects in Drosophila and HeLa cells, insoluble TDP-43 levels and mitochondrial localization of TDP$43^{\mathrm{WT}}$ and TDP- $43^{\mathrm{A} 382 \mathrm{~T}}$ were unaffected in HeLa cells (Fig. 8a, b). In addition, insoluble $\mathrm{CHCHD} 10^{\mathrm{S} 59 \mathrm{~L}}$ and TDP-43 levels were not changed by PINK1 knockdown (Fig. 8c, d), suggesting that PINK1 accumulation in response to $C H C H D 10^{559 \mathrm{~L}}$ expression is parallel or downstream (or both) of the TDP-43 pathway. CHCHD10 ${ }^{W T}$ co-expression with $C H C H D 10^{S 59 L}$ reduced PINK1 accumulation (Fig. 8e). Intriguingly, CHCHD10 ${ }^{W T}$ expression also reduced PINK1 and parkin accumulation caused by mild

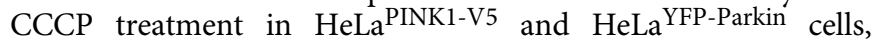
respectively (Fig. 8f and Supplementary Fig. 8a, b). Therefore, CHCHD10 ${ }^{W T}$ protected mitochondria not only through TDP-43 but also by preventing PINK1 accumulation. CHCHD10WT exerted a protective effect through both TDP-43 and PINK1 without modulating $\mathrm{CHCHD} 10^{559 \mathrm{~L}}$ insolubility (Fig. 9), suggesting that augmenting $\mathrm{CHCHD} 10$ expression or activity is also a promising therapeutic strategy, in addition to specifically blocking TDP-43 mitochondrial translocation and/or PINK1 activity.

\section{Discussion}

Since the initial identification of the S59L substitution in CHCHD10, additional variants have been identified and suggested as pathogenic mediators of ALS-FTD, SMAJ, and mitochondrial myopathy. Despite efforts to elucidate their pathogenic mechanisms, many controversial findings suggest that mutations in CHCHD10 do not share a common disease-causing mechanism $1111,12,19-22$. We found that only CHCHD10 ${ }^{559 L}$ induced consistent dominant cell toxicity in both Drosophila and HeLa cells ${ }^{55}$.

Consistent with recent studies demonstrating a dominant mechanism of $\mathrm{CHCHD} 10^{\mathrm{S} 59 \mathrm{~L}}$ in $\mathrm{CHCHD} 10^{\mathrm{KO}}$ mice and CHCHD10 ${ }^{S 59 L}$ knockin mice ${ }^{56,57}$, our own findings do not support simple loss-of-function or haploinsufficiency mechanisms. Woo et al. previously proposed a dominant-negative mechanism of $\mathrm{CHCHD} 10^{\mathrm{S} 9 \mathrm{~L}}$ in Caenorhabditis elegans and mammalian systems. Interestingly, their observations are similar to our findings. However, our data support a dominant gain-of-toxic function mechanism for $C H C H D 10^{S 59 L}$, whereas Woo et al. proposed 
a

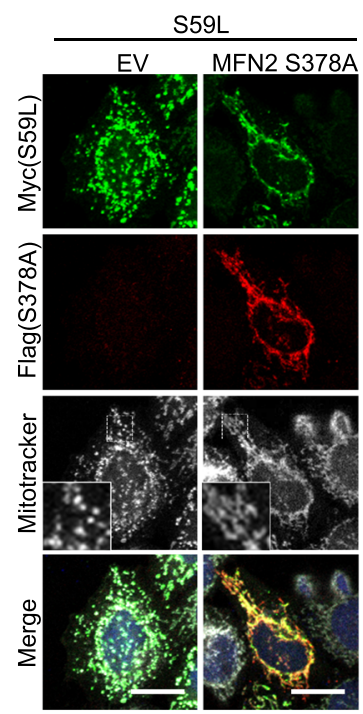

d

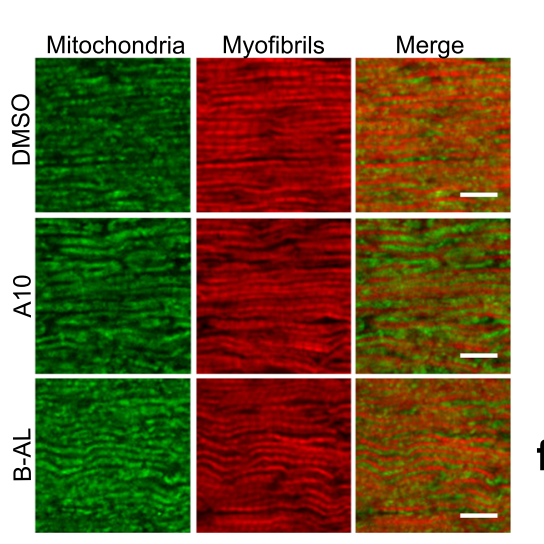

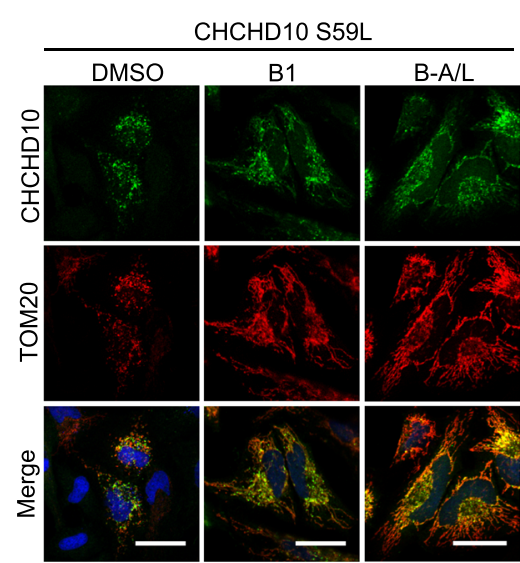

e

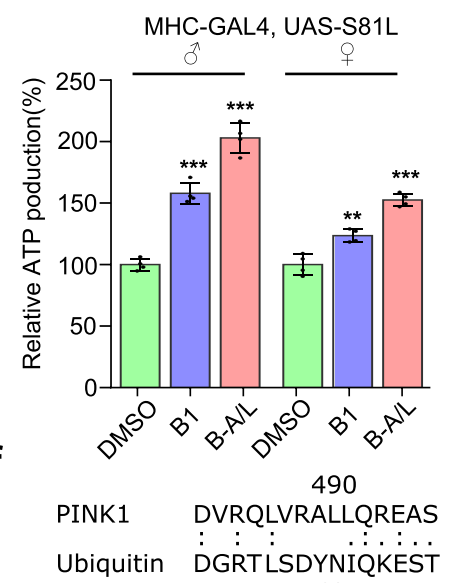

C

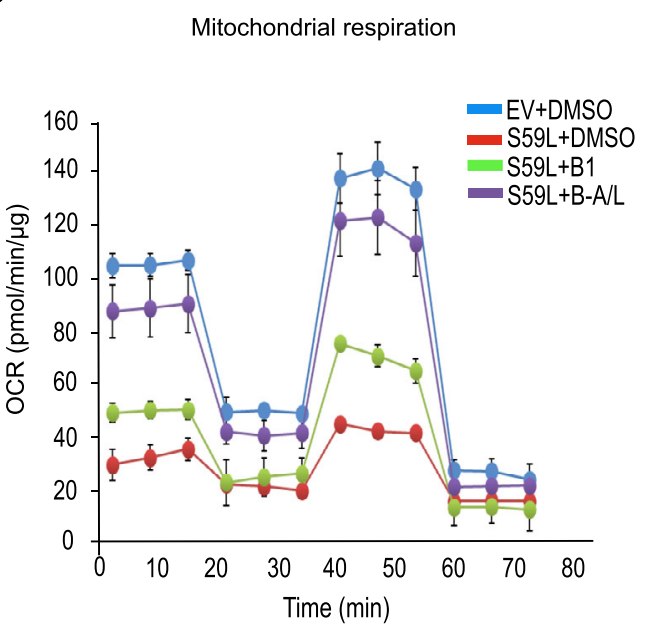

g

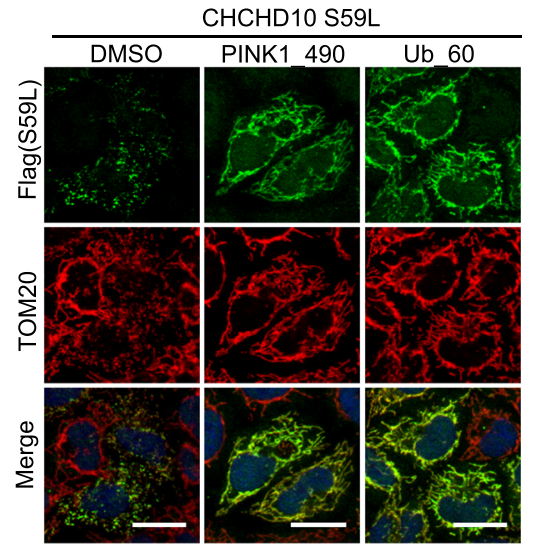

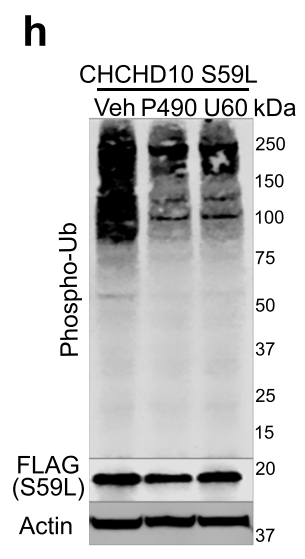

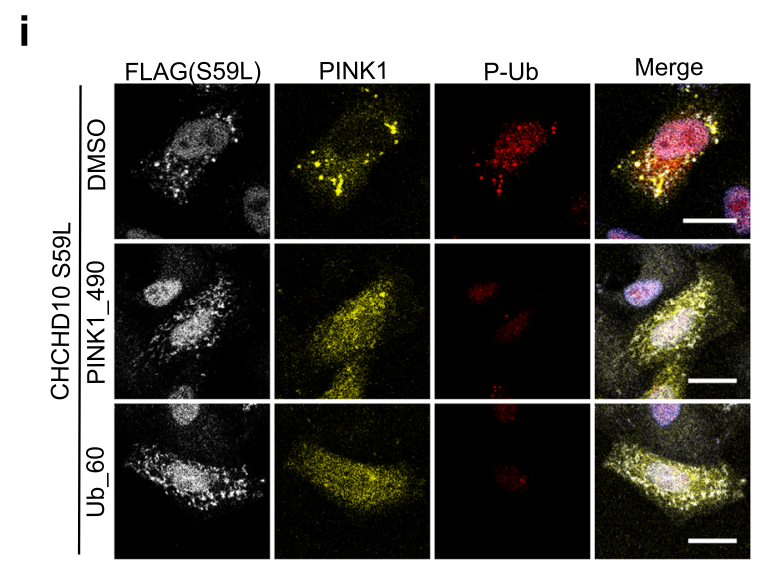

j

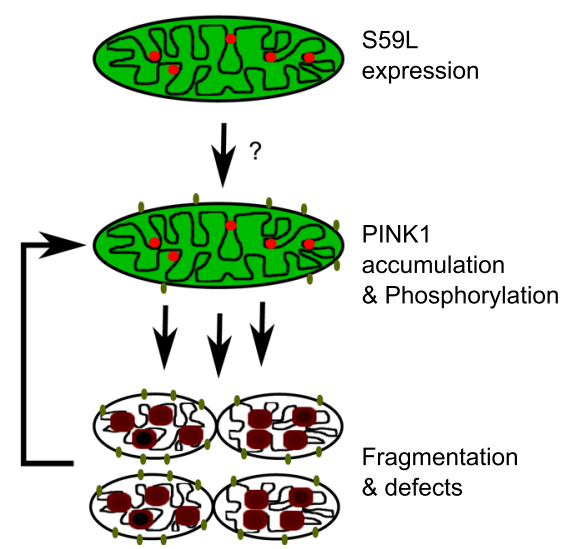

a dominant-negative mechanism, although they also observed results that can support a dominant gain-of-toxic function mechanism. Although these two mechanisms are not necessarily mutually exclusive, several aspects of our findings primarily support a dominant gain-of-toxic function mechanism. First, the Drosophila eye phenotypes driven by $\mathrm{C} 2 \mathrm{C} 10 \mathrm{H}^{559 L}$ did not differ between the $\mathrm{C} 2 \mathrm{C} 10 \mathrm{H}^{\text {null }}$ or $\mathrm{C} 2 \mathrm{C} 10 \mathrm{H}^{W T}$ background, indicating that the mutations exert a dominant gain-of-toxic function rather than suppressing WT activity as a dominant negative. Second, $\mathrm{CHCHD} 10^{\mathrm{S} 59 L}$ is toxic regardless of the presence of $\mathrm{CHCHD} 10^{W T}$ (and $\mathrm{CHCHD}_{2}{ }^{W T}$ ). Third, we confirmed the genetic modifiers modulating the dominant gain-of-toxic function in Drosophila in mammalian cells, as well. Fourth, parkin accumulation did not occur in CHCHD10KO HeLaYFP-Parkin cells, but it did occur with CHCHD $10^{S 59 L}$ expression $^{55}$, indicating that the cell toxicity caused by reduced $\mathrm{CHCHD} 10$ differs from that of $\mathrm{CHCHD} 10^{\mathrm{S} 59 \mathrm{~L}}$. 
Fig. 7 CHCHD10 559 -induced toxicity is mitigated by inhibiting PINK1 activity and modulating its target. a HeLa cells were transfected with Myc-tagged $\mathrm{CHCHD} 10^{S 59 L}$ and FLAG-tagged MFN2 $3378 \mathrm{~A}$. Mitochondria were stained with MitoTracker (deep red FM) and antibodies against Myc and FLAG $24 \mathrm{~h}$ after transfection. White outlined boxes (MitoTracker) were magnified in the left corner. Representative images from three independent experiments are shown. Scale bar $=20 \mu \mathrm{m}$. b HeLa cells were transfected with FLAG-tagged CHCHD10S59L and treated with MFN2 agonists B1 (50 nM) and B-A/L (5 nM) for $24 \mathrm{~h}$. Cells were immunostained with antibodies against FLAG and TOM20. Representative images from three independent experiments are shown. Scale bar $=$ $20 \mu \mathrm{m}$. c Mitochondrial respiration was measured by Seahorse XF Cell Mito Stress tests after mitofusin agonists treatment. A representative graph of a single experiment is shown (mean $\pm \mathrm{SD}$ ). Actual statistical analyses were performed with four independent experiments (see Supplementary Figs. 7e, 9l). d Indirect flight muscles from 10-day-old adult flies fed with B1 and B-A/L (each $10 \mu \mathrm{M}$ ) were stained with streptavidin-Alexa Fluor 488 and phalloidin-Alexa Fluor 594. DMSO was used as a vehicle. Representative images from three independent experiments are shown. Scale bar $=10 \mu \mathrm{m}$. e ATP levels in thoraxes from the indicated genotypes (aged 10 days) were measured. Data are mean \pm SD (one-way ANOVA and post hoc Dunnett test, twosided, ${ }^{\star \star \star} p=0.00013$ and $1.8 \mathrm{e}-05$ in male, ${ }^{\star \star} p=0.0012$ and ${ }^{\star \star \star} p=0.00027$ in female for $\mathrm{B} 1$ and $\mathrm{B}-\mathrm{A} / \mathrm{L}$, respectively, compared to DMSO; $n=4$ independent experiments). f Peptide sequences of two PINK1 inhibitors. $\mathbf{g}$ HeLa cells were treated with PINK1_490 (0.15 $\mu \mathrm{g} / \mathrm{ml})$ or Ub_60 (0.15 $\mu \mathrm{g} / \mathrm{ml})$ and transfected with FLAG-tagged $\mathrm{CHCHD10} 559 \mathrm{~L}$. DMSO was used as a vehicle. Cells were immunostained with antibodies against FLAG and TOM20 $24 \mathrm{~h}$

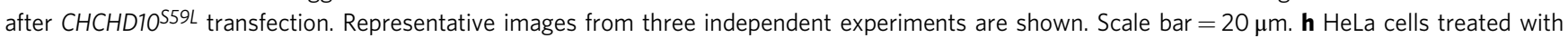
peptide inhibitors and transfected with FLAG-tagged CHCHD10S59L were analyzed with an anti-phospho-ubiquitin antibody. Representative images from three independent experiments are shown. i HeLa cells were treated with peptide inhibitors followed by transfection of FLAG-tagged CHCHD10S59L and YFP-PINK1. The cells were immunostained with antibodies against FLAG and TOM20. Representative images from three independent experiments are shown. Scale bar $=20 \mu \mathrm{m}$. j A schematic depicting the positive feedback mechanism of $C H C H D 10^{S 59 L}$-mediated toxicity through PINK1 accumulation.

Fifth, CHCHD10WT did not accumulate in $\mathrm{CHCHD} 10^{\mathrm{S} 59 \mathrm{~L}}$ aggregate-like structures. Sixth, $\mathrm{CHCHD} 10^{W T}$ overexpression did not affect $\mathrm{CHCHD} 10^{\mathrm{S} 59 \mathrm{~L}}$ aggregate formation and insolubility. Therefore, both $\mathrm{CHCHD} 10^{\mathrm{WT}}$ and $\mathrm{CHCHD} 10^{\mathrm{S} 59 \mathrm{~L}}$ are involved in the same pathway, independently in reverse directions, because $\mathrm{CHCHD} 10$ has a protective role in mitochondria that is also protective for $\mathrm{CHCHD} 10^{S 59 L}$-driven dominant cell toxicity, which occurred independently of disrupting WT activity. However, we cannot completely rule out the contribution of loss-of-functionor dominant-negative-like effects. We anticipate that some effect of reduced WT activity occurs in disease pathogenesis. Our data suggest that the dominant cell toxicity of $\mathrm{CHCHD} 10^{S 59 L}$ can be mitigated by co-expressing similar levels of $\mathrm{CHCHD} 10^{W T}$. Therefore, it is possible that a heterozygous CHCHD10 $559 \dot{L}$ mutation does not induce severe degeneration, as long as WT activity blocks mutant cell toxicity. However, an age-dependent reduction of WT function or a change in the ratio of WT to mutant expression may trigger disease symptoms later. Taken together, multiple or possibly mixed mechanisms may exist, and $\mathrm{CHCHD} 10^{S 59 L}$ may cause a strong dominant phenotype that can be successfully modeled in both Drosophila and HeLa cells.

Our efforts to define the downstream pathways of dominant CHCHD $10^{S 59 L}$-mediated cell toxicity yielded two axes and multiple molecular targets that can be therapeutically modulated (Fig. 9). The mitochondrial translocation of TDP-43 is a toxicitygenerating mechanism in $C H C H D 10^{S 59 L}$-expressing cells. Wang et al. ${ }^{25}$ demonstrated that excess TDP-43 mitochondrial translocation induces mitochondrial dysfunction, and blocking this translocation abolishes mitochondrial toxicity. CHCHD $10^{S 59 L}$ induced TDP-43 mitochondrial translocation and inhibiting this translocation mitigated $\mathrm{CHCHD} 10^{S 59 L_{\text {-induced }} \text { mitochondrial }}$ abnormalities. The association between TDP-43 and CHCHD10 was previously proposed ${ }^{19}$, and Wang et al. raised a question for the importance of the mitochondrial TDP-43. In support of this, we showed that $\mathrm{CHCHD} 10^{\mathrm{S} 59 \mathrm{~L}}$ bound to TDP- 43 more greatly than did $\mathrm{CHCHD} 10 \mathrm{WT}$ and that inhibition of TDP-43 mitochondrial translocation mitigated the $C H C H D 10^{S 59 L}$-induced phenotype. In addition, the effects of $C H C H D 10^{W T}$ on TDP-43 insolubility and translocation generally support that TDP-43 is a key effector generating mitochondrial toxicity in ALS-FTD.

We identified PINK1 and PRKN as strong genetic modifiers of $C 2 C 10 H^{S 81 L}$-mediated cell toxicity. PINK1/parkin-mediated pathways are generally regarded as protective for cells by removing damaged mitochondria. However, CHCHD $10^{S 59 L}$ expression induced PINK1 stabilization in mitochondria, and genetic/pharmacologic inhibition of PINK1 clearly mitigated CHCHD $10^{S 59 L}$-induced cell toxicity. Previous reports showed that reducing PINK1 or parkin-mediated pathways are beneficial in in vivo disease models of SOD1, FUS, and TARDBP mutations ${ }^{58-60}$. We demonstrated that MFN2 agonists enhanced ATP production in flies expressing the GGGGCC repeats of C9ORF72.

Two downstream phosphorylation substrates of PINK1, MFN, and mitofilin mediated $C H C H D 10^{S 59 L}$-induced cell toxicity. Although fusion activity in $C H C H D 10^{S 59 L}$-expressing cells is not altered $^{1}$, our data indicate that PINK1 accumulation and subsequent inactivation of MFN by phosphorylation is responsible for fragmented mitochondria and the respiratory defects caused by $C H C H D 10^{S 59 L}$. An MFN2 agonist developed for CMT2 was also effective for $C H C H D 10^{S 59 L}$-mediated cell toxicity in this context and may also be effective for the $\mathrm{CHCHD} 10^{\mathrm{G} 66 \mathrm{~V}}$ mutation causing SMAJ or CMT2. Overexpression of a PINK1 phosphorylation-null Mitofilin mutant also rescued $C 2 C 10 H^{S 81 L}$ mediated cell toxicity, suggesting that deformation of the MICOS complex was not based on a direct interaction, but through phosphorylation by PINK1. Therefore, the degree of PINK1 stabilization may correspond with the phenotypic severity of mutant CHCHD10.

While this manuscript was under review for publication, two independent studies reported $\mathrm{CHCHD} 10^{\mathrm{S} 59 \mathrm{~L}}$-mediated OMA1 peptidase activation ${ }^{61}$, subsequent degradation of OPA1 resulting in mitochondrial fragmentation ${ }^{61,62}$, and a protective effect of CHCHD10WT against TDP-43 mitochondrial accumulation ${ }^{62}$. Because OMA1 and PINK1 can be activated in the same experimental conditions, elucidating the association between the OMA1-OPA1 pathway and the PINK1-mediated pathway in CHCHD10 ${ }^{S 59 L}$-induced pathogenesis will be worthwhile. Although we did not observe any meaningful protective effects of CHCHD10WT against toxic TDP-43 $337 \mathrm{~V}$ in Drosophila, this may be due to the strong overexpression of $T D P-43^{M 337 V}$. Therefore, investigating the protective role of CHCHD10 in ALSFTD and other degenerative diseases with mitochondrial defects is important. In contrast with our findings, two studies reported that their findings were also observed in multiple CHCHD2 and CHCHD10 double-knockout models ${ }^{61}$ or by CHCHD10 knockdown in cell culture ${ }^{62}$. These findings combined with our data suggest that $C H C H D 10^{S 59 L}$-induced gain-of-toxic function, partial loss of $C H C H D 10^{W T}$, and dominant-negative-like inhibition of $\mathrm{CHCHD} 2$ may coexist during disease pathogenesis or individually contribute to specific aspects of disease pathogenesis. 
a

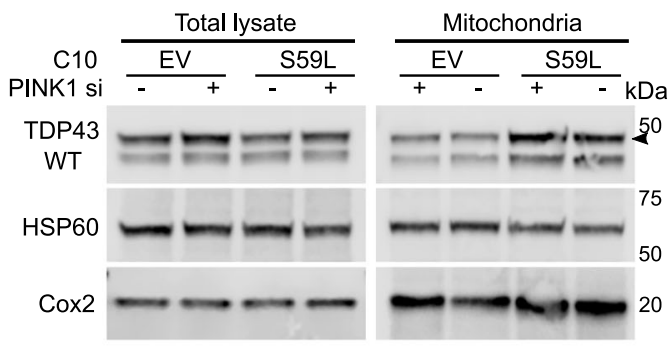

b

Mitochondrial TDP-43

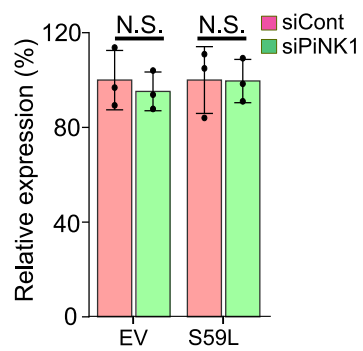

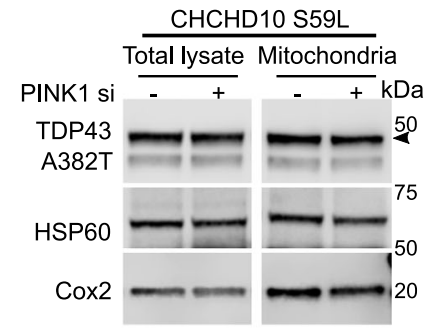

C

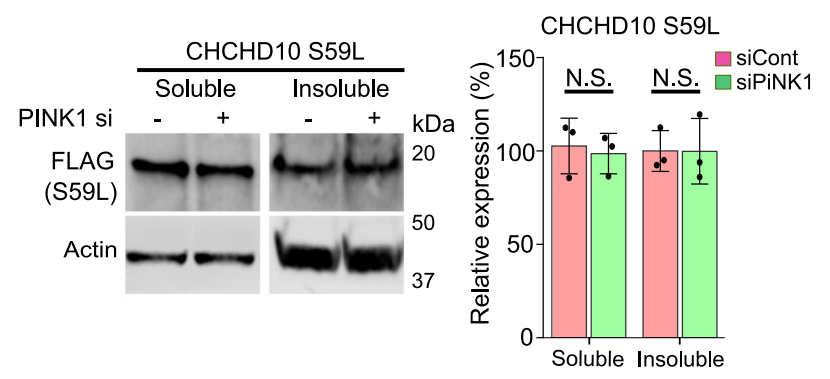

d e

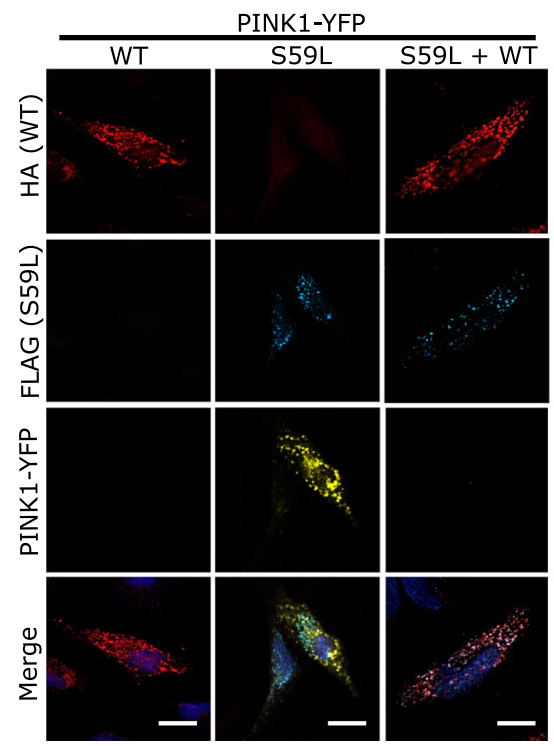

f

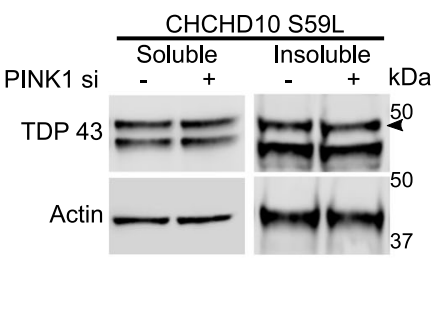

TDP-43 level

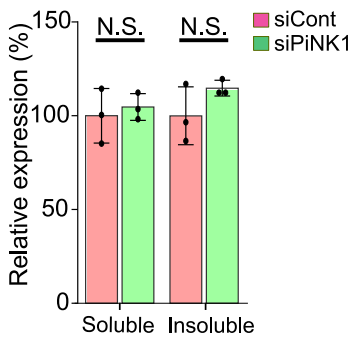

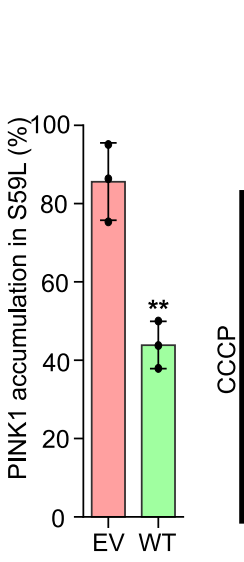
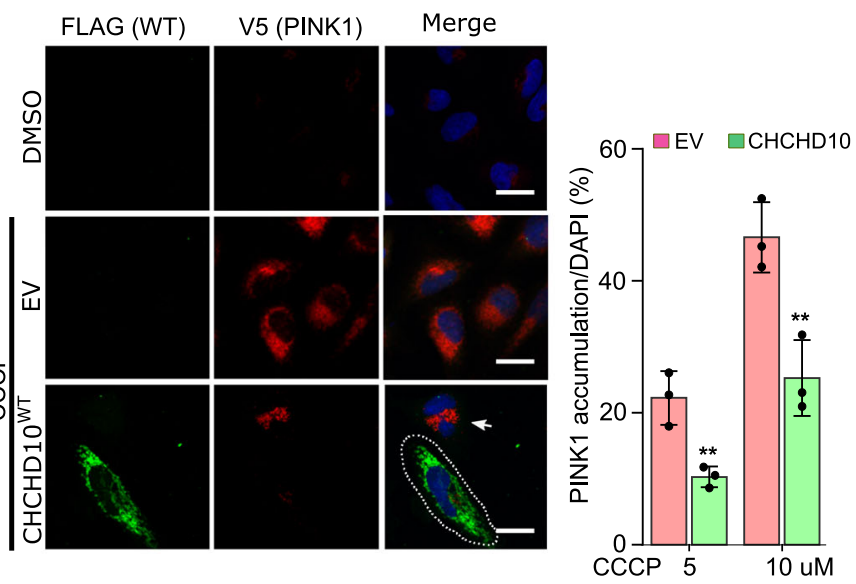

Fig. 8 Dominant toxicity of $\mathbf{C H C H D 1 0}{ }^{559 L}$ is mediated independently by TDP-43 and PINK1 signaling. a HeLa cells transfected with PINK1 siRNA were co-transfected with empty vector (EV) or FLAG-tagged CHCHD10S59L and FLAG-tagged TARDBPWT. Fractionated mitochondria were analyzed with antiTDP-43 (arrowhead indicates transfected TDP-43) or anti-HSP60 and anti-Cox2 (loading controls) antibodies. Data are shown as mean \pm SD (two-sided $t$ test, NS $=$ not significant; $n=4$ independent experiments). b HeLa cells transfected with PINK1 siRNA were co-transfected with empty vector (EV) or FLAG-tagged CHCHD10S59L and TARDBPA382T. Fractionated mitochondria were analyzed with anti-TDP-43 (arrowhead indicates transfected TDP-43) or anti-HSP60 and anti-Cox2 (loading controls) antibodies. c HeLa cells transfected with PINK1 siRNA were transfected with FLAG-tagged CHCHD10S59L RIPA-soluble and insoluble fractions were analyzed with anti-FLAG and anti-actin (loading control) antibodies. Data are shown as mean \pm SD (two-sided $t$ test, NS = not significant; $n=3$ independent experiments). d HeLa cells transfected with PINK1 siRNA were co-transfected with FLAG-tagged CHCHD10S59L and TARDBP. RIPA-soluble and insoluble fractions were analyzed with anti-TDP-43 and anti-actin (loading control) antibodies. Data are shown as mean $\pm \mathrm{SD}$ (two-sided $t$ test, NS $=$ not significant; $n=4$ independent experiments). e HeLa cells transfected with PINK1-YFP, FLAG-tagged CHCHD10 559 , and HA-tagged CHCHD10 WT or EV were visualized with anti-FLAG (blue) and anti-HA (red) antibodies and YFP (yellow) after $24 \mathrm{~h}$ of transfection. PINK1-positive cells with/without $\mathrm{CHCHD10WT}$ in $\mathrm{CHCHD10} 559 \mathrm{~L}$-expressing cells were counted (two-sided $t$ test, ${ }^{\star \star} p=0.00335 ; n=3$ independent experiments, $>50$ cells for each group). Scale bar $=20 \mu \mathrm{m}$. $\mathbf{f}$ HeLaPINK1-V5-His cells transfected with EV or CHCHD10WT were treated with CCCP $(10 \mu \mathrm{M})$ for $6 \mathrm{~h}$. Cells were immunostained with anti-FLAG (green) and anti-V5 (red) antibodies to visualize CHCHD10 and PINK1, respectively. Arrow indicates PINK1 accumulated in a non-transfected cell neighboring a CHCHD10-transfected cell (white dashed line). The percentage of PINK1positive cells from the empty vector (EV) or CHCHD10WT-transfected cells were calculated after 5 or $10 \mu \mathrm{M} \mathrm{CCCP}$ treatment for $6 \mathrm{~h}$. Data are shown as mean \pm SD (two-sided $t$ test, ${ }^{\star \star} p=0.009055$ for $5 \mu \mathrm{M}$ and 0.009203 for $10 \mu \mathrm{M} \mathrm{CCCP}$ treatment; $n=3$ independent experiments, $>200$ cells for each group). Scale bar $=20 \mu \mathrm{m}$. 


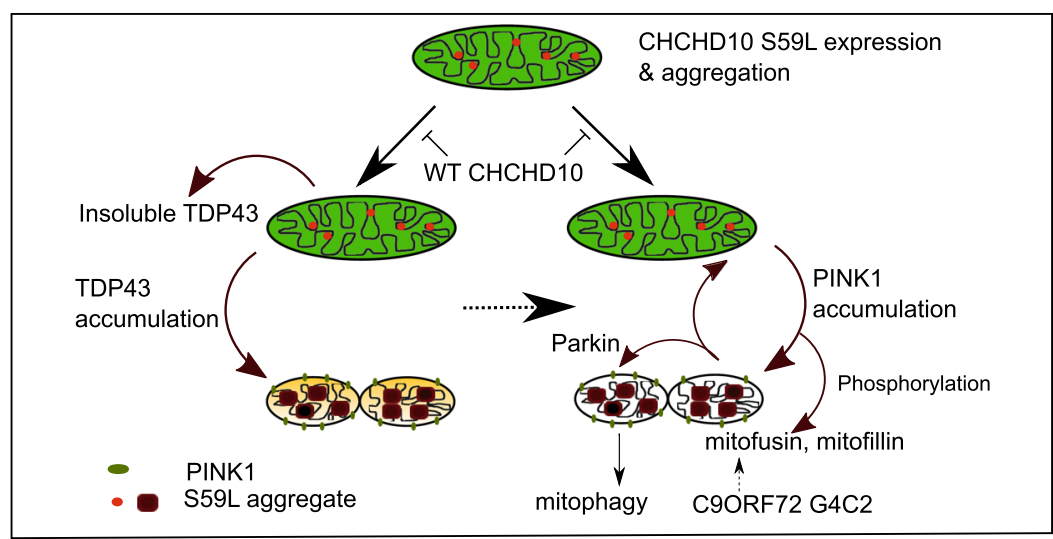

Fig. 9 Two axes of $\mathbf{C H C H D 1 0}^{\text {S59L-induced toxicity. a } \mathrm{CHCHD10}}{ }^{\mathrm{S} 59 \mathrm{~L}}$ can generate cellular toxicity with or without WT $\mathrm{CHCHD10}$ and its close paralog $\mathrm{CHCHD} 2$. The toxicity of the gain-of-function $\mathrm{CHCHD10} 559 \mathrm{~L}$ mutant is mediated by two different pathways. TDP-43 axis: Upon $\mathrm{CHCHD10S59L}$ expression, TDP-43 becomes more insoluble in the cytoplasm, probably due to mitochondria-induced stresses 56,57 and accumulates in mitochondria via increased binding with $\mathrm{CHCHD} 10^{559 L}$. The toxicity generated by mitochondrial TDP-43 can be mitigated by inhibitors for TDP-43 mitochondrial translocation. PINK1

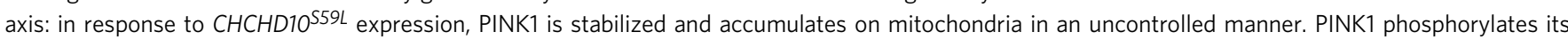
downstream targets, including mitofusin, mitofilin, and Parkin, which results in significant mitochondrial fragmentation, maladaptive cristae formation, and excessive mitophagy. A positive feedback mechanism via PINK1 accumulation may accelerate these processes. Genetic or chemical modulation of this pathway can mitigate $\mathrm{CHCHD10}$ S59L-induced cellular toxicity and C9orf72 G4C2 repeat-induced degeneration. Interestingly, WT CHCHD10 expression reduces $\mathrm{CHCHD10}$ S59L-mediated cellular toxicity through both TDP-43 and PINK1 axes.

Augmenting $\mathrm{CHCHD} 10^{\mathrm{WT}}$ activity may be a promising therapeutic strategy, regardless of specific CHCHD10 mutations. $\mathrm{CHCHD} 10^{W T}$ expression is increased in response to various stresses $^{63}$. We and others observed that $\mathrm{CHCHD} 10^{W T}$ expression not only rescued mutant phenotypes but also increased mitochondrial length and respiratory activity when it was expressed alone in both Drosophila and HeLa cells. Although the mechanism by which CHCHD10 enhances mitochondrial function and reduced PINK1-mediated cell toxicity is not clear, our findings support that pharmacologic or epigenetic augmentation of CHCHD10 expression may mitigate such mitochondrial defects.

\section{Methods}

Chemicals and peptides. CCCP was purchased from Sigma-Aldrich. The MFN agonists $\mathrm{B} 1$ and $\mathrm{B} / \mathrm{A}-\mathrm{L}$ were described previously $\mathrm{y}^{51}$. ScPM and PM1 peptides for TDP-43 were kindly provided by Xinglong Wang. Pink1_490 and Ub_60 peptides were synthesized by Thermo Scientific with N-terminal TAMRA 5/6 and Cterminal amidation.

DNA constructs. All complementary DNAs (cDNAs) for human CHCHD10 ${ }^{W T}$ and variants were synthesized and inserted in the pcDNA3 vector containing a FLAG, HA, or Myc tag by Genescripts. mTagRFP-T-Mito-7 (\#58023) ${ }^{64}$, TDP43tdTOMATO-HA (\#28205) ${ }^{65}$, EGFP-LC3 (\#21073) ${ }^{66}$, mCherry-LC3B $(\# 40827)^{67}$, pEYFP-C1-DRP1 $(\# 45160)^{68}$, MFN2-YFP $(\# 28010)^{69}$, pEYFP-N1PINK1 $(\# 101874)^{70}$, and LAMP1-mGFP (\#34831) ${ }^{71}$ plasmids were obtained from Addgene. FLAG-MFN2 ${ }^{W T}$, FLAG-MFN2 $2^{S 378 A}$, and FLAG-MFN2 ${ }^{S 378 D}$ were described previously ${ }^{51}$. TARDBP ${ }^{W T}$, TARDBPG298S, TARDBPA315T, and TARDB$P^{A 382 T}$ plasmids were gifts from Xinglong Wang. Mito-QC (pBabe.hygro-mcherryGFP fis 101-152) was provided by Ian Ganley.

RNA extraction, CDNA synthesis, and quantitative reverse transcription-PCR (qRT-PCR). Total RNAs were extracted from patient fibroblasts using TRIzol reagent (Thermo Fisher Scientific). Prior to reverse transcription, residual genomic DNA was removed with DNase I (Thermo Fisher Scientific). The cDNA was then reverse transcribed using transcription first-strand cDNA Synthesis Kit (Roche Applied Science) with $1 \mu \mathrm{g}$ total RNA and oligo-dT as primer. All PCRs were performed in triplicate. QRT-PCR was carried out using SYBR Green Master Mix (Roche Applied Science) on a Light Cycler LC480. Results were normalized to $\beta-2$ macroglubulin or $28 \mathrm{~S}$ genes. All primer sequences are included in Supplementary Table 2 .

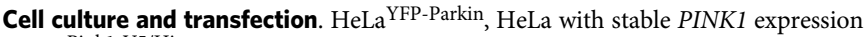

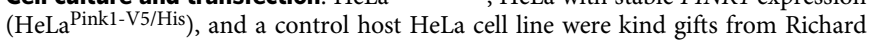

Youle. PINK1 ${ }^{K O}$ HeLa cells and a matched control HeLa cell line were kindly provided by Wade Harper. HEK293T and SH-SY5Y cells were purchased from ATCC. Cells were maintained in culture in Dulbecco's modified Eagle's medium (Gibco) or DMEM/F12 50/50 (Corning) supplemented with 10\% fetal bovine serum (Gibco), $1 \times$ penicillin/streptomycin (Invitrogen), and GlutaMax-1× (Gibco). Cells were transfected using FuGENE6 transfection reagent (Promega), Lipofectamine 3000 (Invitrogen), or jetPrime (Polyplus). RNAi-mediated knockdown of target genes was performed by transfection of the ON-TARGET plus-SMART pool siRNAs (Dharmacon) by using Lipofectamine RNAiMAX (Invitrogen) for the following genes: non-targeting control, PINK1, MFN1, MFN2, OPTN, NDP52, and DRP1. Pink1_490 and Ub_60 peptides were delivered by using PULSin protein delivery reagent (Polyplus).

CRISPR/Cas9-mediated gene editing and generation of cell lines. Each two plasmid vectors [pSpCas9(BB)-2A-Puro (XP459) V2.0] containing a single guide RNA (sgRNA) oligomer for CHCHD10 and CHCHD2 targeting near the Nterminal region were purchased from GenScript. Sequences for the sgRNA targeting the N-terminal of $\mathrm{CHCHD} 10$ are as follows: 5'-GCCTCGGGGAAGCCGCAGCG-3' and $5^{\prime}$-GCCGCGCTGCGGCTTCCCCG- $3^{\prime}$ and for the CHCHD2 are as follows: $5^{\prime}$-CCGAAGCCGCACCTCCCGCA- $3^{\prime}$ and $5^{\prime}$ - CTCAGATGAGAGCTGCACCC-3'. The plasmids were transfected into HeLa and HeLa YFP-Parkin cells with jetPrime reagent. After $24 \mathrm{~h}$, the transfected cells were detached with trypsin and plated individually into 96-well round-bottom plates. Cells were then expanded, and single clones were analyzed by immunofluorescence and immunoblotting to screen protein levels in CHCHD10 knockout and CHCHD10/2 double knockout.

Antibodies and immunoblotting. The following primary antibodies were used: FLAG (Sigma and Proteintech, 1:1000), HA (Cell Signaling and Proteintech, 1:1000), Myc (Proteintech, 1:1000), CHCHD10 (Proteintech, 1:1000), CHCHD2 (Proteintech, 1:1000), TOM20 (Cell Signaling and Santa Cruz Biotechnology, 1:1000), TDP-43 (Proteintech and Santa Cruz Biotechnology, 1:1000), HSP60 (Cell Signaling Technology, 1:1000), COX2 (Abcam, 1:2000), LC3B (Cell Signaling Technology, 1:1000), phospho-ubiquitin (EMD Millipore, 1:1000), Actin (Santa Cruz Biotechnology and Proteintech, 1:3000), PINK1 (Santa Cruz Biotechnology and Novus, 1:1000), DRP1 (Cell Signaling Technology, 1:1000), MFN1 (Cell Signaling Technology, 1:1000), MFN2 (Cell Signaling Technology, 1:1000), NDP52 (Proteintech, 1:1000), and OPTN (Proteintech, 1:1000). Samples were collected and lysed in RIPA buffer (Cell Signaling Technology) containing a protease inhibitor cocktail (Sigma) and subsequently separated by sodium dodecyl sulfatepolyacrylamide gel electrophoresis (SDS-PAGE) after measuring protein concentration by bicinchoninic acid (BCA) (Pierce). Immunoblots were visualized and analyzed with the Odyssey FC System (LI-COR).

Co-immunoprecipitation. HEK293T cells transfected FLAG-tagged CHCHD10 WT or S59L and TDP-43-tomato-HA or HA-tagged CHCHD10 WT or S59L and 
TDP-43-FLAG were solubilized with NP-40 lysis buffer (20 mM Tris, $137 \mathrm{mM}$ $\mathrm{NaCl}, 1 \% \mathrm{NP}-40,2 \mathrm{mM}$ EDTA with protein inhibitor cocktail). After sonication on ice, lysates were collected by centrifuge. An equal amount of protein lysates were incubated with anti-FLAG M2 or anti-HA affinity gel (Sigma) overnight at $4{ }^{\circ} \mathrm{C}$, washed three times with phosphate-buffered saline (PBS) containing $1 \%$ Tween-20, and then resuspended the pellet with $2 \times$ LDS sample buffer. The precipitates were subjected SDS-PAGE and analyzed with the Odyssey FC System (LI-COR).

Solubility and biochemical analyses. Transfected cells were washed twice with PBS, lysed in cold RIPA buffer [50 mM Tris ( $\mathrm{pH} 7.5$ ), $150 \mathrm{mM} \mathrm{NaCl}, 1 \%$ Triton X$100,0.5 \%$ sodium deoxycholate, $0.1 \%$ SDS, and $1 \mathrm{mM} \mathrm{EDTA}$ ) and sonicated on ice. Cellular lysates cleared by ultracentrifugation at $100,000 \times g$ for $30 \mathrm{~min}$ at $4{ }^{\circ} \mathrm{C}$ to prepare RIPA-soluble fractions. RIPA-insoluble pellets were washed twice with protease inhibitors in cold PBS, sonicated, and recentrifuged. RIPA-insoluble proteins were extracted with urea buffer [ $7 \mathrm{M}$ urea, $2 \mathrm{M}$ thiourea, $4 \%$ CHAPS $30 \mathrm{mM}$ Tris ( $\mathrm{pH}$ 8.5)], sonicated, and centrifuged at $100,000 \times g$ for $30 \mathrm{~min}$ at $22{ }^{\circ} \mathrm{C}$. Supernatants from the first centrifugation were analyzed for soluble fractions. Total protein in each sample was measured by the BCA method and resolved on ExpressPlus PAGE 4-20\% gels (GenScript).

Cellular and mitochondrial fractionation. Mitochondria were isolated from cells with a Mitochondrial Isolation Kit (Thermo Scientific) by using Dounce homogenizers. A mixture of cytosolic and mitochondrial fractions was obtained after low-speed centrifugation at $500 \times g$ for $15 \mathrm{~min}$ at $4^{\circ} \mathrm{C}$. Mitochondrial-enriched pellets were collected at $3000 \times g$ for $10 \mathrm{~min}$ at $4{ }^{\circ} \mathrm{C}$. Cytosolic supernatants were obtained and cleared by centrifugation at $12,000 \times g$ for $10 \mathrm{~min}$ at $4{ }^{\circ} \mathrm{C}$. Crosscontamination between fractions was analyzed with anti-tubulin and anti-HSP60 antibodies for each cytosolic and mitochondrial compartment.

Mitochondrial respiratory activity assay. Mitochondrial respiration in HeLa, $C H C H D 10^{K O} \mathrm{HeLa}, C H C H D 2 / 10^{D K O} \mathrm{HeLa}$, HeLa YFP-Parkin, and $C H C H D 10^{K O}$ HeLa ${ }^{\text {YFP-Parkin }}$ cells were measured by using the Seahorse Extracellular Flux Analyzer XFp (Agilent Technologies) with the XF Cell Mito Stress Test Kit (Agilent Technologies). Transfected cells $\left(1 \times 10^{4}\right)$ were counted using ADAM-MC2 (NanoEntek) and plated into V3-PS 96-well plates the day before performing the assay. Assay media were supplemented with $1 \mathrm{mM}$ pyruvate, $2 \mathrm{mM}$ glutamine, and $15 \mathrm{mM}$ glucose. Standard mitochondria stress tests were performed by first measuring basal values followed by measurements after sequential addition of $1 \mu \mathrm{M}$ oligomycin, $0.5 \mu \mathrm{M}$ FCCP, and $0.5 \mu \mathrm{M}$ rotenone/antimycin A. After the assay, protein concentrations of each well were determined via BCA assay and used to normalize oxygen consumption rate values. The Seahorse assay parameters and experimental outcomes are summarized in Supplementary Table 3.

Immunofluorescence staining and imaging. The following primary antibodies were used: FLAG (Sigma and Proteintech, 1:200), FLAG-Alexa Fluor 488 (Invitrogen, 1:250), CHCHD10 (Proteintech, 1:200), CHCHD2 (Proteintech, 1:200), V5 (Life Technology, 1:200), TOM20 (Cell Signaling and Santa Cruz Biotechnology, 1:250), TDP-43 (Proteintech, 1:200), LAMP1 (Cell Signaling Technology, 1:200), and phospho-ubiquitin (EMD Millipore, 1:100). Cells were plated on 4-well chamber slides (Lab-Tek), fixed with 4\% paraformaldehyde in PBS (EMS Millipore), permeabilized with $0.1 \%$ Triton X-100, and blocked with $5 \%$ bovine serum albumin (BSA) in PBS. Primary antibodies were diluted in 5\% BSA in PBS and incubated overnight at $4{ }^{\circ} \mathrm{C}$. Samples were then rinsed three times with PBSTween-20 and incubated with secondary antibodies for $1.5 \mathrm{~h}$ at room temperature. Coverslips were mounted onto microscope slides with Prolong Diamond Antifade Reagent with DAPI (4',6-diamidino-2-phenylindole) (Invitrogen). Samples were observed with an LSM 710 confocal microscope (Carl Zeiss) or Nikon Crest X-light 2 spinning disc confocal microscope (Nikon). Co-localization was analyzed with co-localization tool of ZEN software (Carl Zeiss). For patient primary fibroblast imaging, cells were incubated in a $100 \mathrm{nM}$ solution of MitoTracker red (Invitrogen) for $15 \mathrm{~min}$ at $37^{\circ} \mathrm{C}$. The medium was replaced by DMEM supplemented with $10 \%$ FBS and penicillin $(100 \mathrm{U} / \mathrm{ml}) /$ streptomycin $(0.1 \mathrm{mg} / \mathrm{ml})$ and cells were incubated for $2 \mathrm{~h}$ at $37^{\circ} \mathrm{C}$, washed in PBS, and then fixed. Specimens were analyzed using a Zeiss LSM880 confocal laser-scanning microscope. The images were deconvolved with Huygens Essential Software (Scientific Volume Imaging). The deconvolved images were used for quantitative mitochondrial network analysis with Huygens Essential Software. Mitochondrial network length was quantified for 48-116 randomly selected individual cells.

Generation of Drosophila lines. To generate transgenic Drosophila lines carrying $\mathrm{C} 2 \mathrm{C} 10 \mathrm{H}$, codon-optimized $\mathrm{CHCHD10}$, and their variants, all cDNAs were synthesized with or without a C-terminal FLAG tag and cloned into pUASTattB by GenScript. Transgenic Drosophila lines were generated by BestGene with standard ФC31 integrase-mediated transgenesis into the attP2 site on chromosome 3 (locus $68 \mathrm{~A} 4$ ), the attP40 site on chromosome 2 (locus 25C6), or the VK27 site on chromosome 3 (locus 89E11).
Drosophila genetics. Fly cultures and crosses were performed on standard fly food (Genesee Scientific) and raised at $25^{\circ} \mathrm{C}$. The GMR-GAL4 and OK371-GAL4 drivers were obtained from the Bloomington Stock Center. MHC-GAL4 was a gift from Guillermo Marques. All tested RNAi, deficiency, duplication, and classical allele lines are listed in Supplementary Table 1.

Drosophila neuronal phenotype analysis. To quantify the synaptic bouton numbers of Drosophila larvae ${ }^{44}$, ten third-instar larvae for each group were collected and pinned on Sylgard dishes with tungsten pins. After dissecting the dorsal sides of larvae and removing the trachea and internal organs, the larvae were fixed with $4 \%$ paraformaldehyde in PBS. Larvae were stained with a presynaptic antihorseradish peroxidase antibody (1:200, Jackson Immunoresearch) and postsynaptic anti-discs large antibody (1:200, Developmental Studies Hybridoma Bank). The NMJs at muscle 4 were used for all analyses.

Drosophila behavioral assay. Six to seven wandering third-instar larvae from each group were collected, washed, and placed onto a $3 \%$ agarose gel in a $10-\mathrm{cm}$ dish Larval crawling was recorded by a digital camera for $30 \mathrm{~s}$. Moving distances of individual larva were tracked and measured using Tracker software (Open Source Physics, 5.0.7). To test flight ability, 20 flies from each group were funneled into a $500-\mathrm{ml}$ glass cylinder. The distribution of flies in the cylinder was recorded by a digital camera, and the average scores from five independent experiments were calculated.

Drosophila immunoblotting. Heads of 2-day-old adults were prepared and ground in LDS sample buffer by using a motor-driven plastic pestle homogenizer and centrifuged at $16,000 \times g$ for $10 \mathrm{~min}$. Supernatants were boiled for $10 \mathrm{~min}$ and analyzed by immunoblotting with the Odyssey FC system (LI-COR). Proteins were separated with 4-20\% ExpressPlus PAGE Gels (GenScript), transferred onto nitrocellulose membranes, and probed with anti-FLAG. Anti-actin antibody was used as a loading control.

Drosophila adult muscle preparation and immunohistochemistry. To assess the mitochondrial morphology and sarcomere structure of the indirect flight muscles ${ }^{44}$, dissected adult flies were fixed with $4 \%$ paraformaldehyde in PBS for 1 $\mathrm{h}$, embedded in OCT compound (Fisher Scientific), and frozen with liquid nitrogen or dry ice. Samples were sectioned by a cryomicrotome (Leica). After fixing with 4\% paraformaldehyde in PBS, samples were permeabilized with $0.2 \%$ Triton X-100 buffer in PBS and blocked with 5\% BSA solution in PBS for $1 \mathrm{~h}$. Samples were incubated with streptavidin-Alexa Fluor 488 (Invitrogen) and phalloidin-Alexa Fluor 594 (Invitrogen) overnight at $4{ }^{\circ} \mathrm{C}$ for mitochondrial and muscle staining, respectively. Samples were mounted with Prolong Diamond Antifade Reagent with DAPI and imaged with an LSM 710 confocal microscope (Carl Zeiss) with $\times 63$ magnification.

Drosophila ATP assay. Fly thoraxes from each group were collected and homogenized in $20 \mu \mathrm{l}$ of homogenization buffer [100 mM Tris, $4 \mathrm{mM}$ EDTA, and $6 \mathrm{M}$ guanidine-HCL $(\mathrm{pH} 7.8)]$ and centrifuged at $16,000 \times g$ for $10 \mathrm{~min}$. The supernatants were diluted 1:200 and 1:10 with deionized water and subjected to ATP concentration and protein concentration measurements, respectively. ATP concentration was determined by using the CellTiter Glo Luminescent Cell Viability Assay Kit (Promega) and normalized to total protein.

Image analysis and statistical analysis. Mitochondrial branch length was measured by using the MiNA toolset combined with the ImageJ software ${ }^{72}$. Statistical analysis was performed with Prism5 (GraphPad) software. For Drosophila eye quantification, we implemented ilastik, which is an interactively supervised machine learning-based tool for various bioimage analysis to classify ommatidia in fly eye images ${ }^{73}$. Then we used Flynotyper ${ }^{31}$, which is a computational tool for assessing the Drosophila eye morphological defects, to calculate phenotypic scores $(P$-scores), which indicate the irregularity of ommatidial arrangement. Eye images for group A (Fig. 1b, Supplementary Fig. 1d, Fig. 3a, Supplementary Fig. 3d, and Fig. 5a) were captured with a Leica M205C stereomicroscope equipped with a ring light and a Leica DFC320 digital camera. Eye images for group B (Fig. 1c, Fig. 3f, Supplementary Fig. 3a, e, and Supplementary Fig. 5b, 7a, b) were captured with a Nikon SMZ1500 stereomicroscope equipped with a ring light and a Nikon DXM1200 digital camera. Although we applied the same criteria to analyze all eye images with ilastik and Flynotyper, the $P$-scores can be compared only within each experiment because of differences in the equipment, setting, and researchers. For statistical analyses of Seahorse Assays, $Z$-scores were calculated using OCR values from each plate. $Z$-score $=(\mathrm{OCR}$ value - mean of OCR values $) /$ standard deviation of OCR values. Student's $t$ test (two-tailed), analysis of variance (ANOVA), and post hoc analysis (Tukey's or Dunnett's tests, two-tailed) were used to test statistical significance. All $p$ values $\leq 0.05$ were considered statistically significant. 
Reporting summary. Further information on research design is available in the Nature Research Reporting Summary linked to this article.

\section{Data availability}

All data supporting the findings of this study are provided within the paper and its Supplementary information. Additional information, Drosophila, and cell lines generated in this study are available upon request from N.C.K. Requests for mitofusin agonists should be made to G.W.D. Source data are provided with this paper.

Received: 24 October 2019; Accepted: 3 March 2021;

Published online: 26 March 2021

\section{References}

1. Bannwarth, S. et al. A mitochondrial origin for frontotemporal dementia and amyotrophic lateral sclerosis through CHCHD10 involvement. Brain 137, 2329-2345 (2014).

2. Zhou, Z. D., Saw, W. T. \& Tan, E. K. Mitochondrial CHCHD-containing proteins: physiologic functions and link with neurodegenerative diseases. Mol. Neurobiol. 54, 5534-5546 (2017).

3. Mejzini, R. et al. ALS genetics, mechanisms, and therapeutics: where are we now? Front. Neurosci. 13, 1-27 (2019).

4. Johnson, J. O. et al. Mutations in the CHCHD10 gene are a common cause of familial amyotrophic lateral sclerosis. Brain 137, e311 (2014).

5. Müller, K. et al. Two novel mutations in conserved codons indicate that $\mathrm{CHCHD} 10$ is a gene associated with motor neuron disease. Brain 137, e309 (2014).

6. Zhang, M. et al. Mutation analysis of $\mathrm{CHCHD} 10$ in different neurodegenerative diseases. Brain 138, e380 (2015).

7. Van Rheenen, W., Diekstra, F. P., Van Den Berg, L. H. \& Veldink, J. H. Are CHCHD10 mutations indeed associated with familial amyotrophic lateral sclerosis? Brain 137, e313 (2014)

8. Chiò, A. et al. CHCH10 mutations in an Italian cohort of familial and sporadic amyotrophic lateral sclerosis patients. Neurobiol. Aging 36, 1767.e3-1767.e6 (2015).

9. Ronchi, D. et al. CHCHD10 mutations in Italian patients with sporadic amyotrophic lateral sclerosis. Brain 138, e372 (2015).

10. Chaussenot, A. et al. Screening of CHCHD10 in a French cohort confirms the involvement of this gene in frontotemporal dementia with amyotrophic lateral sclerosis patients. Neurobiol. Aging 35, 2884.e1-2884.e4 (2014).

11. Genin, E. C. et al. CHCHD 10 mutations promote loss of mitochondrial cristae junctions with impaired mitochondrial genome maintenance and inhibition of apoptosis. EMBO Mol. Med. 8, 58-72 (2016).

12. Marroquin, N. et al. Screening for CHCHD10 mutations in a large cohort of sporadic ALS patients: no evidence for pathogenicity of the p.P34S variant. Brain 139, e8 (2016)

13. Wong, C. H. et al. The CHCHD10 P34S variant is not associated with ALS in a UK cohort of familial and sporadic patients. Neurobiol. Aging 36, 2908. e17-2908.e18 (2015).

14. Ajroud-Driss, S. et al. Mutation in the novel nuclear-encoded mitochondrial protein CHCHD10 in a family with autosomal dominant mitochondrial myopathy. Neurogenetics 16, 1-9 (2014).

15. Penttilä, S. et al. Late onset spinal motor neuronopathy is caused by mutation in CHCHD10. Ann. Neurol. 77, 163-172 (2015).

16. Auranen, M. et al. CHCHD10 variant p.(Gly66Val) causes axonal CharcotMarie-Tooth disease. Neurol. Genet. 1, e1 (2015).

17. Watts, G. D. J. et al. Inclusion body myopathy associated with Paget disease of bone and frontotemporal dementia is caused by mutant valosin-containing protein. Nat. Genet. 36, 377-381 (2004).

18. Senderek, J. et al. Autosomal-dominant distal myopathy associated with a recurrent missense mutation in the gene encoding the nuclear matrix protein, matrin 3. Am. J. Hum. Genet. 84, 511-518 (2009).

19. Woo, J. A. A. et al. Loss of function CHCHD10 mutations in cytoplasmic TDP-43 accumulation and synaptic integrity. Nat. Commun. 8, 1-15 (2017).

20. Brockmann, S. J. et al. CHCHD10 mutations p. R15L and p. G66V cause motoneuron disease by haploinsufficiency. Hum. Mol. Genet. 27, 706-715 (2018).

21. Straub, I. R. et al. Loss of CHCHD10-CHCHD2 complexes required for respiration underlies the pathogenicity of a CHCHD10 mutation in ALS. Hum. Mol. Genet. 27, 178-189 (2018).

22. Genin, E. C. et al. Loss of MICOS complex integrity and mitochondrial damage, but not TDP-43 mitochondrial localisation, are likely associated with severity of CHCHD10-related diseases. Neurobiol. Dis. 119, 159-171 (2018).
23. Higgins, C. M. J., Jung, C., Ding, H. \& Xu, Z. Mutant Cu, Zn superoxide dismutase that causes motoneuron degeneration is present in mitochondria in the CNS. J. Neurosci. 22, 1-6 (2002).

24. Mattiazzi, M. et al. Mutated human SOD1 causes dysfunction of oxidative phosphorylation in mitochondria of transgenic mice. J. Biol. Chem. 277, 29626-29633 (2002).

25. Wang, W. et al. The inhibition of TDP-43 mitochondrial localization blocks its neuronal toxicity. Nat. Med. 22, 869-878 (2016).

26. Lopez-Gonzalez, R. et al. Poly(GR) in C9ORF72-related ALS/FTD compromises mitochondrial function and increases oxidative stress and DNA damage in iPSC-derived motor neurons. Neuron 92, 383-391 (2016).

27. Kiskinis, E. et al. Pathways disrupted in human ALS motor neurons identified through genetic correction of mutant SOD1. Cell Stem Cell 14, 781-795 (2014).

28. Magrané, J., Cortez, C., Gan, W. B. \& Manfredi, G. Abnormal mitochondrial transport and morphology are common pathological denominators in SOD1 and TDP43 ALS mouse models. Hum. Mol. Genet. 23, 1413-1424 (2014).

29. $\mathrm{Hu}, \mathrm{Y}$. et al. An integrative approach to ortholog prediction for diseasefocused and other functional studies. BMC Bioinformatics 12, 357 (2011).

30. Cavallaro, G. Genome-wide analysis of eukaryotic twin CX9C proteins. Mol. Biosyst. 6, 2459-2470 (2010).

31. Iyer, J. et al. Quantitative assessment of eye phenotypes for functional genetic studies using Drosophila melanogaster. G3 Genes Genomes Genet. 6, 1427-1437 (2016).

32. Noble, G. P., Dolph, P. J. \& Supattapone, S. Interallelic transcriptional enhancement as an in vivo measure of transvection in Drosophila melanogaster. G3 Genes Genomes Genet. 6, 3139-3148 (2016).

33. Burstein, S. R. et al. In vitro and in vivo studies of the ALS-FTLD protein $\mathrm{CHCHD} 10$ reveal novel mitochondrial topology and protein interactions. Hum. Mol. Genet. 27, 160-177 (2018).

34. Huang, X. et al. CHCHD2 accumulates in distressed mitochondria and facilitates oligomerization of CHCHD10. Hum. Mol. Genet. 27, 3881-3900 (2018).

35. Prelich, G. Gene overexpression: uses, mechanisms, and interpretation. Genetics 190, 841-854 (2012).

36. Meng, H. et al. Loss of Parkinson's disease-associated protein CHCHD2 affects mitochondrial crista structure and destabilizes cytochrome c. Nat. Commun. 8, 1-18 (2017).

37. Hasegawa, M. et al. Phosphorylated TDP-43 in frontotemporal lobar degeneration and amyotrophic lateral sclerosis. Ann. Neurol. 64, 60-70 (2008).

38. Mackenzie, I. R. A., Rademakers, R. \& Neumann, M. TDP-43 and FUS in amyotrophic lateral sclerosis and frontotemporal dementia. Lancet Neurol. 9, 995-1007 (2010).

39. Chang, J. C. \& Morton, D. B. Drosophila lines with mutant and wild type human TDP-43 replacing the endogenous gene reveals phosphorylation and ubiquitination in mutant lines in the absence of viability or lifespan defects. PLoS ONE 12, 1-24 (2017).

40. Ruan, L. et al. Cytosolic proteostasis through importing of misfolded proteins into mitochondria. Nature 543, 443-446 (2017).

41. Huang, P., Yu, T. \& Yoon, Y. Mitochondrial clustering induced by overexpression of the mitochondrial fusion protein Mfn2 causes mitochondrial dysfunction and cell death. Eur. J. Cell Biol. 86, 289-302 (2007).

42. Jin, S. M. \& Youle, R. J. The accumulation of misfolded proteins in the mitochondrial matrix is sensed by PINK1 to induce PARK2/Parkin-mediated mitophagy of polarized mitochondria. Autophagy 9, 1750-1757 (2013).

43. Jin, S. M. \& Youle, R. J. PINK1-and Parkin-mediated mitophagy at a glance. J. Cell Sci. 125, 795-799 (2012).

44. Kim, N. C. et al. VCP is essential for mitochondrial quality control by PINK1/ Parkin and this function is impaired by VCP mutations. Neuron 78, 65-80 (2013).

45. Koyano, F. et al. Ubiquitin is phosphorylated by PINK1 to activate parkin. Nature 510, 162-166 (2014).

46. Gegg, M. E. et al. Mitofusin 1 and mitofusin 2 are ubiquitinated in a PINK1/ parkin-dependent manner upon induction of mitophagy. Hum. Mol. Genet. 19, 4861-4870 (2010).

47. Ma, P., Yun, J., Deng, H. \& Guo, M. Atg1-mediated autophagy suppresses tissue degeneration in pink1/parkin mutants by promoting mitochondrial fission in Drosophila. Mol. Biol. Cell 29, 3082-3092 (2018).

48. Bhide, S. et al. Increasing autophagy and blocking Nrf2 suppress laminopathyinduced age-dependent cardiac dysfunction and shortened lifespan. Aging Cell 17, 1-14 (2018).

49. Marceau, K. et al. The ubiquitin kinase PINK1 recruits autophagy receptors to induce mitophagy. Nature 57, 742-768 (2015).

50. Tsai, P. I. et al. PINK1 phosphorylates MIC60/Mitofilin to control structural plasticity of mitochondrial crista junctions. Mol. Cell 69, 744-756.e6 (2018). 
51. Rocha, A. G. et al. MFN2 agonists reverse mitochondrial defects in preclinical models of Charcot-Marie-Tooth disease type 2A. Science 360, 336-341 (2018).

52. Freibaum, B. D. et al. GGGGCC repeat expansion in C9orf72 compromises nucleocytoplasmic transport. Nature 525, 129-133 (2015).

53. Kemp, B. E., Pearson, R. B. \& House, C. M.Pseudosubstrate-based peptide inhibitors. Methods Enzymol. 201, 287-304 (1991).

54. Madeira, F. et al. The EMBL-EBI search and sequence analysis tools APIs in 2019. Nucleic Acids Res. 47, W636-W641 (2019).

55. Baek, M., Choe, Y.-J., Dorn, G., Taylor, J. P. \& Kim, N. C. Dominant toxicity of ALS-FTD-associated CHCHD10 S59L is mediated by TDP-43 and PINK1. Preprint at bioRXiv https://doi.org/10.1101/753558 (2019).

56. Anderson, C. J. et al. ALS/FTD mutant CHCHD10 mice reveal a tissuespecific toxic gain-of-function and mitochondrial stress response. Acta Neuropathol. 138, 103-121 (2019).

57. Genin, E. C. et al. Mitochondrial defect in muscle precedes neuromuscular junction degeneration and motor neuron death in CHCHD10 S59L/+ mouse. Acta Neuropathol. 138, 123-145 (2019).

58. Chen, Y. et al. PINK1 and Parkin are genetic modifiers for FUS-induced neurodegeneration. Hum. Mol. Genet. 25, 5059-5068 (2016).

59. Sun, X. et al. Distinct multilevel misregulations of Parkin and PINK1 revealed in cell and animal models of TDP-43 proteinopathy. Cell Death Dis. 9, 953 (2018).

60. Palomo, G. M. et al. Parkin is a disease modifier in the mutant SOD 1 mouse model of ALS. EMBO Mol. Med. 10, e8888 (2018).

61. Liu, Y. T. et al. Loss of CHCHD2 and CHCHD10 activates OMA1 peptidase to disrupt mitochondrial cristae phenocopying patient mutations. Hum. Mol. Genet. 29, 1547-1567 (2020)

62. Liu, T. et al. CHCHD10-regulated OPA1-mitofilin complex mediates TDP-43induced mitochondrial phenotypes associated with frontotemporal dementia. FASEB J. https://doi.org/10.1096/fj.201903133rr, 1-17 (2020).

63. Purandare, N., Somayajulu, M., Hüttemann, M., Grossman, L. I. \& Aras, S. The cellular stress proteins CHCHD10 and MNRR1 (CHCHD2): partners in mitochondrial and nuclear function and dysfunction. J. Biol. Chem. 293, 6517-6529 (2018).

64. Day, R. N. \& Davidson, M. W. The fluorescent protein palette: tools for cellular imaging. Chem. Soc. Rev. 38, 2887-2921 (2009).

65. Yang, C. et al. The C-terminal TDP-43 fragments have a high aggregation propensity and harm neurons by a dominant-negative mechanism. PLOS ONE 5, e15878 (2010).

66. Kabeya, Y. et al. LC3, a mammalian homolog of yeast Apg8p, is localized in autophagosome membranes after processing. EMBO J. 22, 4577 (2003); erratum 19, 5720-5728 (2000).

67. Jahreiss, L., Menzies, F. M. \& Rubinsztein, D. C. The itinerary of autophagosomes: from peripheral formation to kiss-and-run fusion with lysosomes. Traffic 9, 574-587 (2008).

68. Frank, S. et al. The role of dynamin-related protein 1, a mediator of mitochondrial fission, in apoptosis. Dev. Cell 1, 515-525 (2001).

69. Karbowski, M. et al. Spatial and temporal association of Bax with mitochondrial fission sites, Drp1, and Mfn2 during apoptosis. J. Cell Biol. 159, 931-938 (2002).

70. Narendra, D. P. et al. PINK1 is selectively stabilized on impaired mitochondria to activate Parkin. PLoS Biol. 8, e1000298 (2010).

71. Falcón-Pérez, J. M., Nazarian, R., Sabatti, C. \& Dell'Angelica, E. C. Distribution and dynamics of Lamp1-containing endocytic organelles in fibroblasts deficient in BLOC-3. J. Cell Sci. 118, 5243-5255 (2005).

72. Valente, A. J., Maddalena, L. A., Robb, E. L., Moradi, F. \& Stuart, J. A. A simple ImageJ macro tool for analyzing mitochondrial network morphology in mammalian cell culture. Acta Histochem. 119, 315-326 (2017).
73. Berg, S. et al. Ilastik: Interactive Machine Learning for (Bio)Image Analysis. Nat. Methods 16, 1226-1232 (2019).

\section{Acknowledgements}

We thank the Bloomington Drosophila Stock Center, the VDRC Stock Center for fly lines, the IRCAN's Molecular and Cellular Core Imaging (PICMI) facility, and the Research Instrumentation Lab at the University of Minnesota Duluth for assistance. We also thank undergraduate volunteers, Sierra Skudlarek, Austin Kurtz, Anna Huy, and Maddie Chalmers, helping in Drosophila maintenance and plasmids preparation. This work was supported by grants from the Muscular Dystrophy Association, the Wallin neuroscience discovery fund, the Engebretson Drug Design and Discovery Grant, and the NINDS/NIA 1R56NS112296-01 to N.C.K.

\section{Author contributions}

M.B., Y.-J.C, and N.C.K. conceived the project and performed experiments. S.B. performed all experiments using patient-derived fibroblasts. S.M. performed experiments measuring mitochondrial activity. M.B., Y.-J.C., S.B., S.M., J.H.K., V.P.-F., and N.C.K. analyzed data and wrote the manuscript. G.W.D. and J.P.T. provided guidance and helpful insights into design experiments and analyzed the data.

\section{Competing interests}

G.W.D. is the scientific founder of Mitochondria in Motion, Inc., which has license from WUSTL and is commercializing small-molecule mitofusin agonists for the treatment of neurodegenerative diseases. The remaining authors declare no competing interests.

\section{Additional information}

Supplementary information The online version contains supplementary material available at https://doi.org/10.1038/s41467-021-22145-9.

Correspondence and requests for materials should be addressed to N.C.K.

Peer review information Nature Communications thanks John Elrod and the other anonymous reviewer(s) for their contribution to the peer review of this work. Peer reviewer reports are available.

Reprints and permission information is available at http://www.nature.com/reprints

Publisher's note Springer Nature remains neutral with regard to jurisdictional claims in published maps and institutional affiliations.

Open Access This article is licensed under a Creative Commons Attribution 4.0 International License, which permits use, sharing, adaptation, distribution and reproduction in any medium or format, as long as you give appropriate credit to the original author(s) and the source, provide a link to the Creative Commons license, and indicate if changes were made. The images or other third party material in this article are included in the article's Creative Commons license, unless indicated otherwise in a credit line to the material. If material is not included in the article's Creative Commons license and your intended use is not permitted by statutory regulation or exceeds the permitted use, you will need to obtain permission directly from the copyright holder. To view a copy of this license, visit http://creativecommons.org/ licenses/by/4.0/

(c) The Author(s) 2021, corrected publication 2021 